\title{
MAGNETIC DIFFUSIVITY TENSOR AND DYNAMO EFFECTS IN ROTATING AND SHEARING TURBULENCE
}

\author{
A. Brandenburg, ${ }^{1}$ K.-H. RÄDler, ${ }^{2}$ M. Rheinhardt, ${ }^{2}$ And P. J. KäPylä ${ }^{1,3}$ \\ Received 2007 October 22; accepted 2007 November 29
}

\begin{abstract}
The turbulent magnetic diffusivity tensor is determined in the presence of rotation or shear. The question is addressed whether dynamo action from the shear-current effect can explain large-scale magnetic field generation found in simulations with shear. For this purpose a set of evolution equations for the response to imposed test fields is solved with turbulent and mean motions calculated from the momentum and continuity equations. The corresponding results for the electromotive force are used to calculate turbulent transport coefficients. The diagonal components of the turbulent magnetic diffusivity tensor are found to be very close together, but their values increase slightly with increasing shear and decrease with increasing rotation rate. In the presence of shear, the sign of the two off-diagonal components of the turbulent magnetic diffusion tensor is the same and opposite to the sign of the shear. This implies that dynamo action from the shear-current effect is impossible, except perhaps for high magnetic Reynolds numbers. However, even though there is no alpha effect on the average, the components of the $\alpha$ tensor display Gaussian fluctuations around zero. These fluctuations are strong enough to drive an incoherent alpha-shear dynamo. The incoherent shearcurrent effect, on the other hand, is found to be subdominant.
\end{abstract}

Subject headings: MHD — turbulence

Online material: color figures

\section{INTRODUCTION}

Many of the stellar and planetary magnetic fields are believed to be the result of a dynamo process that converts kinetic energy from turbulent motions and shear into magnetic energy. A particular challenge consists in explaining the field on length scales that exceed the scale of the turbulence. This topic has traditionally been addressed within the framework of mean-field electrodynamics (Krause \& Rädler 1980).

Over the decades, the applicability of this theory has repeatedly been questioned (e.g., Piddington 1981; Vainshtein \& Cattaneo 1992). Meanwhile, direct simulations of hydromagnetic turbulence have begun to show dynamo action (Meneguzzi et al. 1981; Meneguzzi \& Pouquet 1989; Nordlund et al. 1992; Brandenburg et al. 1996; Cattaneo 1999). In some particular cases, large-scale fields are being generated (Glatzmaier \& Roberts 1995; Brandenburg et al. 1995; Brandenburg 2001), which raises the question about the mechanism responsible for this phenomenon. In cases where the flow is systematically non-mirror symmetric, the association with an $\alpha$ effect is obvious. However, there are now also examples of nonhelical large-scale dynamos owing to turbulence under the influence of shear alone (Brandenburg 2005a; Yousef et al. 2007). Their interpretation is not straightforward, because several possible mechanisms have been proposed that might produce dynamo action from turbulence and shear alone, i.e., without rotation and stratification that otherwise would have been the main ingredients of an $\alpha$ effect. The most detailed investigations have been carried out in connection with the socalled shear-current effect (Rogachevskii \& Kleeorin 2003, 2004; Rädler \& Stepanov 2006; Rüdiger \& Kitchatinov 2006). Another possibility is a magnetic $\alpha$ effect that is driven by a current helicity flux, as was suggested by Vishniac \& Cho (2001; see also Brandenburg \& Subramanian 2005c). A third possibility might

\footnotetext{
${ }_{1}^{1}$ NORDITA, Roslagstullsbacken 23, SE-10691 Stockholm, Sweden.

2 Astrophysical Institute Potsdam, An der Sternwarte 16, D-14482 Potsdam, Germany.

3 Observatory, University of Helsinki, P.O. Box 14, FI-00014 Helsinki, Finland.
}

be an incoherent (random) $\alpha$ effect with zero mean and finite variance, suggested by Vishniac \& Brandenburg (1997) in connection with accretion disks (see also Sokolov 1997; Silant'ev 2000; Fedotov et al. 2006; Proctor 2007). The only reliable way to determine what is the dominant effect is to calculate all relevant components of the $\alpha$ and turbulent magnetic diffusivity tensors in a general expansion of the electromotive force in terms of the mean magnetic field.

The case considered in Brandenburg (2005a) is unnecessarily complicated, because the shear employed there depends on two Cartesian coordinates. A simpler possibility is to consider a shear flow depending linearly on only one coordinate, and we shall pursue this idea in the present paper. The shear-current effect and the incoherent $\alpha$ effect could then still operate. Because we will use periodic boundary conditions, there can be no magnetic helicity flux, so the Vishniac \& Cho (2001) effect is then ruled out, even though it could still, at least in principle, explain the generation of a mean magnetic field in the simulations of Brandenburg (2005a), which do possess a helicity flux.

In this paper we calculate all relevant components of $\alpha_{i j}$ and $\eta_{i j k}$ using the so-called test field method. This method was introduced by Schrinner et al. $(2005,2007)$ in connection with convection in a spherical shell and used later by Brandenburg (2005b), Sur et al. (2008), and Brandenburg et al. (2008) in connection with forced turbulence in Cartesian boxes. The essence of this method consists in solving evolution equations for the fluctuations of the magnetic field around suitably defined test fields such that all relevant coefficients can be computed.

\section{BASIC ASSUMPTIONS AND METHOD}

In the following we introduce first the mean electromotive force and its relation to the mean magnetic field. We then discuss the equations describing the turbulent flow that eventually leads to this electromotive force and explain the test field method used to calculate the coefficients which relate it to the mean field. Particular attention is paid to the possibility that the shear-current effect may lead to self-excitation of mean magnetic fields. 


\subsection{The Turbulent Electromotive Force}

In mean-field electrodynamics, the behavior of the mean magnetic field $\overline{\boldsymbol{B}}$ depends crucially on the mean electromotive force $\overline{\mathcal{E}}=\overline{\boldsymbol{u} \times \boldsymbol{b}}$, where $\boldsymbol{u}$ and $\boldsymbol{b}$ denote the deviations of the fluid velocity $\boldsymbol{U}$ and the magnetic field $\boldsymbol{B}$ from their mean parts $\overline{\boldsymbol{U}}$ and $\overline{\boldsymbol{B}}$, respectively. For sufficiently weak variations of $\overline{\boldsymbol{B}}$ in space and time and if there is no small-scale dynamo producing a mean electromotive force on its own, we have

$$
\overline{\mathcal{E}}_{i}=\alpha_{i j} \bar{B}_{j}+\eta_{i j k} \partial \bar{B}_{j} / \partial x_{k}
$$

with tensors $\alpha_{i j}$ and $\eta_{i j k}$ determined by $\boldsymbol{u}$ and $\overline{\boldsymbol{U}}$.

In this section it is sufficient to define mean quantities like $\overline{\mathcal{E}}$ or $\overline{\boldsymbol{B}}$, referring to Cartesian coordinates $(x, y, z)$, simply by averaging over all $x$ and $y$. Below, in $\S 2.3$ a different definition is introduced that covers and refines this simple one. Clearly, $\bar{B}$ can now no longer depend on $x$ and $y$, and hence, all its first-order derivatives can be expressed by the components of $\nabla \times \overline{\boldsymbol{B}}=$ $\left(-\partial \bar{B}_{y} / \partial z, \partial \bar{B}_{x} / \partial z, 0\right)$. Slightly deviating from the usual notation, in which $\nabla \times \boldsymbol{B}$ is equal to $\mu_{0} \boldsymbol{J}$, where $\mu_{0}$ is the vacuum permeability, we put in this paper simply $\nabla \times \boldsymbol{B}=\boldsymbol{J}$, being aware that $\boldsymbol{J}$ is then no longer exactly the electric current density. Instead of equation (1) we may now write

$$
\overline{\mathcal{E}}_{i}=\alpha_{i j} \bar{B}_{j}-\eta_{i j} \bar{J}_{j}
$$

with a new tensor $\eta_{i j}$ defined such that $\eta_{i 1}=\eta_{i 23}$ and $\eta_{i 2}=-\eta_{i 13}$. As $\bar{J}_{3}=0$, the $\eta_{i 3}$ are without interest and we may put them equal to zero.

We further consider the background turbulence, which occurs in the absence of rotation or shear, as homogeneous, isotropic, and mirror symmetric. Then we have even under the influence of rotation or shear $\alpha_{i j}=0$; see Appendix A.

As for $\eta_{i j}$, consider first the case of rotation of the fluid with an angular velocity $\Omega$, which defines the Coriolis and centrifugal forces and is assumed to be aligned with the $z$-axis. The actual turbulence is then again homogeneous but no longer isotropic. Instead, it is axisymmetric with respect to the $z$-axis, that is, all mean quantities depending on the turbulent velocity field are invariant under arbitrary rotations about the $z$-axis. We may then conclude by usual symmetry arguments that

$$
\eta_{i j}=\eta_{0} \delta_{i j}+\delta \epsilon_{i j k} \hat{\Omega}_{k}+\delta^{\prime} \hat{\Omega}_{i} \hat{\Omega}_{j}
$$

where $\hat{\boldsymbol{\Omega}}=\boldsymbol{\Omega} / \Omega$ with $\Omega=|\boldsymbol{\Omega}|$, and $\eta_{0}, \delta$, as well as $\delta^{\prime}$ are spatially constant coefficients, which may depend on $\Omega$. So we arrive at

$$
\overline{\mathcal{E}}=-\eta_{0} \overline{\boldsymbol{J}}+\delta \hat{\mathbf{\Omega}} \times \overline{\boldsymbol{J}}
$$

Since $\bar{J}_{3}=0$, the $\delta^{\prime}$ term in equation (3) is without influence. The last term in equation (4) describes the $\Omega \times \bar{J}$ effect (Rädler 1969). Whereas $\eta_{0}$ approaches a nonzero value as $\Omega \rightarrow 0$ (the value determined by the background turbulence), $\delta$ vanishes like $\Omega$. Note that $\overline{\mathcal{E}}_{z}=0$.

Consider next the case with shear defined by the velocity $\boldsymbol{U}^{S}=(0, S x, 0)$. Now the actual turbulence is again homogeneous but no longer axisymmetric. In view of the application of symmetry arguments we consider $\boldsymbol{U}^{S}$ first in the more general (coordinate-independent) form $\boldsymbol{S g}(\boldsymbol{h} \cdot \boldsymbol{x})$, where $\boldsymbol{g}$ and $\boldsymbol{h}$ are unit vectors which are orthogonal to each other and $\boldsymbol{x}$ is the position vector. The only available construction elements for $\eta_{i j}$ are then $\delta_{k l}, \epsilon_{k l m}, \boldsymbol{g}$, and $\boldsymbol{h}$, for due to the homogeneity of the turbulence, $\eta_{i j}$ cannot depend on $\boldsymbol{x}$. Thus, we have

$$
\eta_{i j}=\eta_{0} \delta_{i j}+\kappa_{1} g_{i} g_{j}+\kappa_{2} h_{i} h_{j}+\kappa_{3} g_{i} h_{j}+\kappa_{4} g_{j} h_{i}+\ldots,
$$

where $\eta_{0}, \kappa_{1}, \kappa_{2}, \kappa_{3}$, and $\kappa_{4}$ are spatially constant coefficients and the dots stand for additional terms containing $\epsilon_{k l m}$. The aforementioned coefficients may depend on $S$. (A dependence on scalars defined by $\boldsymbol{g}$ and $\boldsymbol{h}$ is without interest, since $\boldsymbol{g}^{2}=\boldsymbol{h}^{2}=1$ and $\boldsymbol{g} \cdot \boldsymbol{h}=0$.) The terms containing $\epsilon_{k l m}$ have structures like $\epsilon_{i j k} g_{k}$ or $g_{i} \epsilon_{j k l} g_{k} h_{l}$. Since $\boldsymbol{S g}(\boldsymbol{h} \cdot \boldsymbol{x})$ is invariant under simultaneous sign changes of $\boldsymbol{g}$ and $\boldsymbol{h}, \eta_{i j}$ must also have this property and so these terms have to be canceled. Returning now to $\boldsymbol{U}^{S}=$ $(0, S x, 0)$, that is, $\boldsymbol{g}=(0,1,0)$ and $\boldsymbol{h}=(1,0,0)$, we see that

$$
\overline{\mathcal{E}}=-\eta_{0} \overline{\boldsymbol{J}}-\kappa \cdot \overline{\boldsymbol{J}}
$$

with

$$
\boldsymbol{\kappa}=\left(\begin{array}{ccc}
\kappa_{11} & \kappa_{12} & 0 \\
\kappa_{21} & \kappa_{22} & 0 \\
0 & 0 & 0
\end{array}\right)
$$

This covers the "shear-current effect" (Rogachevskii \& Kleeorin 2003). We may assume that $\eta_{0}$ is independent of $S$ (that is, it is determined by the background turbulence alone). Then $\kappa_{11}$ and $\kappa_{22}$ are even functions of $S$ that vanish like $S^{2}$ as $S \rightarrow 0$, whereas $\kappa_{12}$ and $\kappa_{21}$ are odd functions that vanish like $S$. Again, we have $\overline{\mathcal{E}}_{z}=0$.

In both cases, with rotation or with shear, we may restrict our attention to

$$
\overline{\mathcal{E}}_{i}=-\eta_{i j} \bar{J}_{j}, \quad 1 \leq i, j \leq 2
$$

The four quantities $\eta_{i j}$ are simply related to $\eta_{0}$ and $\delta$, or $\eta_{0}$ and the $\kappa_{i j}$, respectively.

\subsection{Turbulence with Rotation or Shear}

We consider a compressible fluid satisfying an isothermal equation of state. In the absence of rotation or shear, the momentum and continuity equations can be written in the form

$$
\begin{gathered}
\frac{\partial \boldsymbol{U}}{\partial t}=-\boldsymbol{U} \cdot \nabla \boldsymbol{U}-c_{s}^{2} \nabla \ln \rho+\boldsymbol{f}+\boldsymbol{F}_{\mathrm{visc}} \\
\frac{\partial \ln \rho}{\partial t}=-\boldsymbol{U} \cdot \nabla \ln \rho-\nabla \cdot \boldsymbol{U}
\end{gathered}
$$

where $c_{s}$ is the sound speed, here considered as constant, $\rho$ is the mass density, and $f$ is a random forcing function. Furthermore, $\boldsymbol{F}_{\text {visc }}=\rho^{-1} \nabla \cdot 2 \rho \nu \mathbf{S}$ is the viscous force, and $S_{i j}=$ $\frac{1}{2}\left(U_{i, j}+U_{j, i}\right)-\frac{1}{3} \delta_{i j} \nabla \cdot \boldsymbol{U}$ is the traceless rate of strain tensor.

To come as close as possible to the assumptions on the background turbulence adopted above, i.e., homogeneity, isotropy, and mirror symmetry, the forcing function $f$ was specified for a cubic domain of size $L \times L \times L$ as follows. During each time step $f$ is a single transverse (solenoidal) plane wave proportional to $\boldsymbol{k}_{f} \times \hat{\boldsymbol{e}}$, where the wavevector $\boldsymbol{k}_{f}$ is taken randomly from a set of predefined vectors with components being integer multiples of $2 \pi / L$ and moduli in a certain interval around an average value which we simply denote by $k_{f}$, and $\hat{\boldsymbol{e}}$ is an arbitrary random unit vector not aligned with $\boldsymbol{k}_{f}$. The corresponding scale, $2 \pi / k_{f}$, is also referred to as the energy-carrying scale of the turbulence. 
Moreover, the time dependence of $f$ is designed to mimic $\delta$-correlation, which is a simple and commonly used form of random driving (cf. Brandenburg 2001). Nevertheless, owing to inertia, the correlation time of the turbulent velocity is of course finite, even for perfect $\delta$-correlation of the forcing.

As mentioned above, we simulate the turbulence in a finite domain using (shearing-) periodic boundary conditions ( $\S 2.5$ ). Then the background turbulence can be at no instant in a strict sense homogeneous, isotropic, or mirror symmetric. It would approach these properties if the ratio of the size of this domain and the scale of the forcing function (that is, $k_{f} / k_{1}$ with $k_{1}=$ $2 \pi / L)$ became very large. There are, however, practical bounds on this ratio. For moderate values, which we have to accept, the background turbulence approaches the mentioned properties only after averaging over long times. Then, of course, the turbulence appears also as statistically steady. By these reasons, mean quantities, that is, averages over $x$ and $y$, which are derived from the turbulence, show still fluctuations in $z$ and $t$, and these disappear after averaging over sufficiently long time intervals.

When rotation is added, two new terms arise on the righthand side of equation (9), the Coriolis force, $-2 \Omega \times U$, and the centrifugal force, $(\boldsymbol{\Omega} \times \boldsymbol{x}) \times \boldsymbol{\Omega}$. The latter is unimportant for weak compressibility, to which we restrict ourselves in the following, and this term would also not be compatible with periodic boundary conditions, so it is neglected.

Turning now to the case with shear we redefine the velocity $\boldsymbol{U}$ by splitting off the shear term $\boldsymbol{U}^{S}$, that is, $\boldsymbol{U} \rightarrow \boldsymbol{U}+\boldsymbol{U}^{S}$. This implies

$\boldsymbol{U} \cdot \nabla \boldsymbol{U} \rightarrow \boldsymbol{U} \cdot \nabla \boldsymbol{U}+\boldsymbol{U}^{S} \cdot \nabla \boldsymbol{U}+\boldsymbol{U} \cdot \nabla \boldsymbol{U}^{S}+\boldsymbol{U}^{S} \cdot \nabla \boldsymbol{U}^{S}$.

The second term on the right-hand side corresponds to an additional advection with the mean flow and will be subsumed in the definition of an advective derivative,

$$
\mathcal{D} / \mathcal{D} t \equiv \partial / \partial t+S x \partial / \partial y
$$

The third term is equal to $S U_{x} \hat{\boldsymbol{y}}$, where $\hat{\boldsymbol{y}}$ is the unit vector in the $y$-direction. The last term in equation (11) vanishes. Thus, equations (9) and (10) turn into

$$
\begin{gathered}
\frac{\mathcal{D} \boldsymbol{U}}{\mathcal{D} t}=-\boldsymbol{U} \cdot \nabla \boldsymbol{U}-S U_{x} \hat{\boldsymbol{y}}-c_{s}^{2} \nabla \ln \rho+\boldsymbol{f}+\boldsymbol{F}_{\mathrm{visc}} \\
\frac{\mathcal{D} \ln \rho}{\mathcal{D} t}=-\boldsymbol{U} \cdot \nabla \ln \rho-\nabla \cdot \boldsymbol{U}
\end{gathered}
$$

It should be noted that $\boldsymbol{U}$ resulting from these equations is not purely turbulent, but also contains a large-scale flow which provides an additional shear and, therefore, a mean vorticity. This is qualitatively suggestive of an hydrodynamic mean-field effect analogous to the shear-current effect; see Elperin et al. (2003).

In this paper we deal, apart from one exception, with the purely kinematic problem, so there is no Lorentz force in equation (13). In $\S 4.1$ the fully nonlinear problem is considered, and hence, the Lorentz force is included in the momentum equation.

\subsection{Test Field Method}

Proceeding now to consequences of the induction equation we consider primarily the case of shear, in which the fluid velocity is $\boldsymbol{U}+\boldsymbol{U}^{S}$. In the case of rotation we have to put $\boldsymbol{U}^{S}$ equal to zero. We further represent $\boldsymbol{B}$ according to $\boldsymbol{B}=\nabla \times \boldsymbol{A}$ by a vector potential $\boldsymbol{A}$. Uncurling the induction equation and using a suitable gauge transformation of $\boldsymbol{A}$ we find ${ }^{4}$

$$
\frac{\mathcal{D} \boldsymbol{A}}{\mathcal{D} t}=-S A_{y} \hat{\boldsymbol{x}}+\boldsymbol{U} \times \boldsymbol{B}-\eta \boldsymbol{J} .
$$

This equation as well as those derived from it in what follows apply to the case of rotation if $\mathcal{D} / \mathcal{D} t$ is replaced by $\partial / \partial t$ and $S$ is put equal to zero.

Now we define a mean field $\bar{F}$ belonging to the field $F$ as

$$
\bar{F}(x, y, z)=\frac{1}{L^{2}} \int_{-L / 2}^{L / 2} \int_{-L / 2}^{L / 2} F(x+\xi, y+\eta, z) d \xi d \eta
$$

with $L$ as specified above. The following comments on the definition from equation (16) as well as equations (17) and (18) below apply, however, even if $L$ is an arbitrary length, not necessarily related to the domain size. Our definition from equation (16) implies that averaging of $F$ commutes with taking any derivatives of $F$ with respect to $x, y, z$, or $t$, that is, the sequence of these operations can be changed. In what follows we also use the rule $\bar{F} G=\overline{F G}$, which applies exactly if $\bar{F}$ is independent of $x$ and $y$, and has otherwise to be considered as an approximation. It is however only needed in cases in which that independence of $x$ and $y$ can be justified, that is, in which it applies exactly. Clearly, $\bar{F}$ is independent of $x$ and $y$ if $F$ is periodic in $x$ and $y$ with the period length $L$. We note further that, owing to equation (16), we have $\bar{x}=x$, and that therefore $\boldsymbol{U}^{S}$ has to be considered as a mean field.

Taking now the average of equation (15) we obtain

$$
\frac{\mathcal{D} \overline{\boldsymbol{A}}}{\mathcal{D} t}=-S \bar{A}_{y} \hat{\boldsymbol{x}}+\overline{\boldsymbol{U}} \times \overline{\boldsymbol{B}}+\overline{\boldsymbol{u} \times \boldsymbol{b}}-\eta \overline{\boldsymbol{J}} .
$$

In view of the determination of $\overline{\mathcal{E}}=\overline{\boldsymbol{u} \times \boldsymbol{b}}$ we are interested in $\boldsymbol{b}=\nabla \times \boldsymbol{a}$, where $\boldsymbol{a}=\boldsymbol{A}-\overline{\boldsymbol{A}}$. Taking the difference between equations (15) and (17) we obtain

$$
\frac{\mathcal{D} \boldsymbol{a}}{\mathcal{D} t}=-S a_{y} \hat{\boldsymbol{x}}+\overline{\boldsymbol{U}} \times \boldsymbol{b}+\boldsymbol{u} \times \overline{\boldsymbol{B}}+\boldsymbol{u} \times \boldsymbol{b}-\overline{\boldsymbol{u} \times \boldsymbol{b}}-\eta \boldsymbol{j},
$$

where $\boldsymbol{j}=\boldsymbol{J}-\overline{\boldsymbol{J}}$.

In order to determine the quantities $\eta_{i j}$ introduced above we specify $\overline{\boldsymbol{B}}$ in the relevant relations such that it is equal to one of the elements out of a set of test fields, $\overline{\boldsymbol{B}}^{q}$, and denote the corresponding $\overline{\mathcal{E}}, \bar{J}$, etc., by $\overline{\mathcal{E}}^{q}, \overline{\boldsymbol{J}}^{q}$, etc., respectively. Then, in particular, equation (8) turns into

$$
\overline{\mathcal{E}}_{i}^{q}=-\eta_{i j} \bar{J}_{j}^{q}, \quad 1 \leq i, j \leq 2 .
$$

After having calculated the $\overline{\mathcal{E}}^{q}$ numerically for two properly chosen $\overline{\boldsymbol{B}}^{q}$ we may then determine the four $\eta_{i j}$.

For the calculation of the $\overline{\mathcal{E}}^{q}$ we apply equation $(18),{ }^{5}$

$$
\begin{aligned}
\frac{\mathcal{D} \boldsymbol{a}^{q}}{\mathcal{D} t}= & -S a_{y}^{q} \hat{\boldsymbol{x}}+\overline{\boldsymbol{U}} \times \boldsymbol{b}^{q}+\boldsymbol{u} \times \overline{\boldsymbol{B}}^{q} \\
& +\boldsymbol{u} \times \boldsymbol{b}^{q}-\overline{\boldsymbol{u} \times \boldsymbol{b}^{q}}-\eta \boldsymbol{j}^{q} .
\end{aligned}
$$

\footnotetext{
${ }^{4}$ Note that $\boldsymbol{U}^{S} \times \boldsymbol{B}$ can be written as $\left(\boldsymbol{U}^{S} \times \nabla \times \boldsymbol{A}\right)_{i}=U_{j}^{S}\left(A_{j, i}-A_{i, j}\right)$. The second term is an advection term and the first term can be written as $U_{j}^{S} A_{j, i}=$ $-U_{j, i}^{S} A_{j}$ plus a gradient term that can be removed with a gauge transformation. Note also that $U_{j, i}^{S} A_{j}=S A_{y} \hat{x}_{i}$.

${ }^{5}$ In the corresponding eq. (27) of Brandenburg (2005b), the $\bar{U}$ term is incorrect. However, this did not affect his results, because $\bar{U}$ either vanished or it consisted only of a shearing motion that was treated correctly in the code.
} 
Although a mean flow can develop in some of the simulations with shear (see above), this term is still weak and is neglected in the following, i.e., we put $\overline{\boldsymbol{U}}=0$ in equation (20).

As test fields $\overline{\boldsymbol{B}}^{q}$ we may use, e.g., the fields $\overline{\boldsymbol{B}}^{q c}$ defined by

$$
\overline{\boldsymbol{B}}^{1 c}=B(\cos k z, 0,0), \quad \overline{\boldsymbol{B}}^{2 c}=B(0, \cos k z, 0),
$$

with a constant $B$ and a constant wavenumber $k$. Denoting the corresponding $\overline{\mathcal{E}}^{q}$ by $\overline{\mathcal{E}}^{q c}$ we find

$$
\overline{\mathcal{E}}_{i}^{1 c}=\eta_{i 2} B k \sin k z, \quad \overline{\mathcal{E}}_{i}^{2 c}=-\eta_{i 1} B k \sin k z, \quad i=1,2 .
$$

After having calculated the $\overline{\mathcal{E}}^{q c}$, these equations allow us to determine the $\eta_{i j}$. In order to avoid difficulties at the zeros of $\sin k z$, it is useful to carry out the calculations with test fields $\overline{\boldsymbol{B}}^{q s}$, defined analogously to the $\overline{\boldsymbol{B}}^{q c}$ but with $\sin k z$ instead of $\cos k z$. For the corresponding $\overline{\mathcal{E}}^{q s}$ we find then equations analogous to equations (22) but with $-\cos k z$ instead of $\sin k z$. From equations (22) and the equations above we obtain immediately (see also Brandenburg 2005b)

$$
\begin{aligned}
& \eta_{i 1}=-(B k)^{-1}\left(\overline{\mathcal{E}}_{i}^{2 c} \sin k z-\overline{\mathcal{E}}_{i}^{2 s} \cos k z\right), \\
& \eta_{i 2}=(B k)^{-1}\left(\overline{\mathcal{E}}_{i}^{1 c} \sin k z-\overline{\mathcal{E}}_{i}^{1 s} \cos k z\right), \quad i=1,2 .
\end{aligned}
$$

We recall that for homogeneous turbulence, which is considered here, the $\eta_{i j}$ have to be independent of $z$. That is, the cos $k z$ and $\sin \mathrm{kz}$ in equations (22) and (23) should be compensated by $z$-dependences of the $\overline{\mathcal{E}}_{i}^{q c}$ and $\overline{\mathcal{E}}_{i}^{q s}$. However, due to fluctuations (cf. $\S 2.2$ ), no perfect compensation can be expected.

We further recall that in equation (1) and so also in equation (2) all derivatives of $\overline{\boldsymbol{B}}$ that are higher than first order have been ignored. By this reason, the results for the $\eta_{i j}$ obtained with the above test fields apply exactly only in the limit $k \rightarrow 0$. In general, there is a dependence of the $\eta_{i j}$ on $k$. This corresponds to a nonlocal connection between $\overline{\mathcal{E}}$ and $\overline{\boldsymbol{B}}$, which is considered here only in a very weak sense (by taking into account first-order derivatives of $\overline{\boldsymbol{B}}$ ). In a more general sense, it is investigated in Brandenburg et al. (2008). Here we have used $k=k_{1}$, where $k_{1}$ means the smallest finite wavenumber in the $z$-direction in the domain in which the turbulence is simulated, $k_{1}=2 \pi / L$; see $\S 2.5$.

The results for $\eta_{i j}$ are also independent of the value of $B$. If one wanted to address the question of nonlinearity, which is not the purpose of this paper, one must also solve equation (15) and allow the resulting magnetic field to feed back onto the flow via the Lorentz force.

For the discussion of the results concerning $\eta_{i j}$ we introduce the quantities

$$
\eta_{t}=\frac{1}{2}\left(\eta_{11}+\eta_{22}\right), \quad \eta_{T}=\eta+\eta_{t}, \quad \epsilon=\frac{1}{2}\left(\eta_{11}-\eta_{22}\right) .
$$

In the case of rotation we put further

$$
\delta=\frac{1}{2}\left(\eta_{12}-\eta_{21}\right)
$$

and expect $\epsilon$ to be equal to zero, while $\eta_{12}$ and $\eta_{21}$ have to have the same nonzero moduli, but opposite signs so that $\delta$ is nonzero. With shear, however, $\epsilon$ can be in general nonzero, and there is no simple relation between $\eta_{12}$ and $\eta_{21}$.
It is convenient to present $\eta_{t}$ in normalized form and express it in terms of the quantity

$$
\eta_{t 0}=\frac{1}{3} u_{\mathrm{rms}} k_{f}^{-1},
$$

which is the result obtained under the first-order smoothing approximation applied to the high-conductivity limit under the assumption that the correlation time is given by $\left(u_{\mathrm{rms}} k_{f}\right)^{-1}$, i.e., that the Strouhal number is unity (cf. Brandenburg \& Subramanian 2005b).

\subsection{Dispersion Relation}

In the case of rotation without shear, there are only decaying solutions of the mean-field equations. This can be easily seen from the energy balance equation for the mean magnetic field (see Rädler 1980). The situation with shear alone is however different, and the possibility of a so-called shear-current dynamo is still under debate (see Rogachevskii \& Kleeorin 2003, 2004; Brandenburg 2005b; Rädler \& Stepanov 2006; Rüdiger $\&$ Kitchatinov 2006). We look therefore for solutions of equation (17) with $\overline{\boldsymbol{u} \times \boldsymbol{b}}$ specified in the sense of equation (8). Using the Ansatz $\overline{\boldsymbol{A}}=\tilde{\boldsymbol{A}} \exp (\lambda t+i k z)$ with a generally complex $\lambda$ and any real $k$ satisfying $k \ll k_{f}$, we find first

$$
\left(\begin{array}{cc}
\lambda+\left(\eta_{T}+\epsilon\right) k^{2} & \eta_{12} k^{2}+S \\
\eta_{21} k^{2} & \lambda+\left(\eta_{T}-\epsilon\right) k^{2}
\end{array}\right)\left(\begin{array}{c}
\tilde{A}_{x} \\
\tilde{A}_{y}
\end{array}\right)=0 .
$$

The requirement of nonvanishing $\tilde{A}$ poses an eigenvalue problem for $\lambda$. The two eigenvalues, normalized to $\eta_{T} k^{2}$, are

$$
\frac{\lambda_{ \pm}}{\eta_{T} k^{2}}=-1 \pm \frac{1}{\eta_{T}} \sqrt{\left(\frac{S}{k^{2}}+\eta_{12}\right) \eta_{21}+\epsilon^{2}} .
$$

A necessary and sufficient condition for an exponentially growing solution is that the radicand in equation (28) is positive and that it exceeds $\eta_{T}^{2}$. If the $S$ term dominates and the others are neglected, this condition turns into

$$
D_{\eta S} \equiv \frac{S \eta_{21}}{\left(\eta_{T} k\right)^{2}}>1
$$

As $k$ can be made arbitrarily small (by making the domain size large enough), this condition is always satisfiable if only $S \eta_{21}>0$. The neglect of the terms without $S$ in the radicand is justified if $\left|\eta_{12} \eta_{21}+\epsilon^{2}\right| / \eta_{T}^{2} \ll\left|D_{\eta S}\right|$, which can again always be guaranteed by sufficiently small $k$. Under this condition, the maximum growth rate with respect to $k$ is $S \eta_{21} / 4 \eta_{T}$ and occurs at $k=$ $\left(S \eta_{21}\right)^{1 / 2} / 2 \eta_{T}$. Consequently, as long as $\eta_{21}$ can be considered linear in $S$ the maximum growth rate is proportional to $S^{2}$ and the corresponding $k$ is proportional to $S$.

\subsection{Simulations}

For the numerical simulations we use the Pencil Code, ${ }^{6}$ where the test field algorithm has already been implemented. We employ periodic boundary conditions in the $y$ - and $z$-directions and shearing-periodic boundary conditions in the $x$-direction (Wisdom $\&$ Tremaine 1988; Hawley et al. 1995) and use a resolution of

\footnotetext{
${ }^{6}$ See http://www.nordita.org/software/pencil-code.
} 

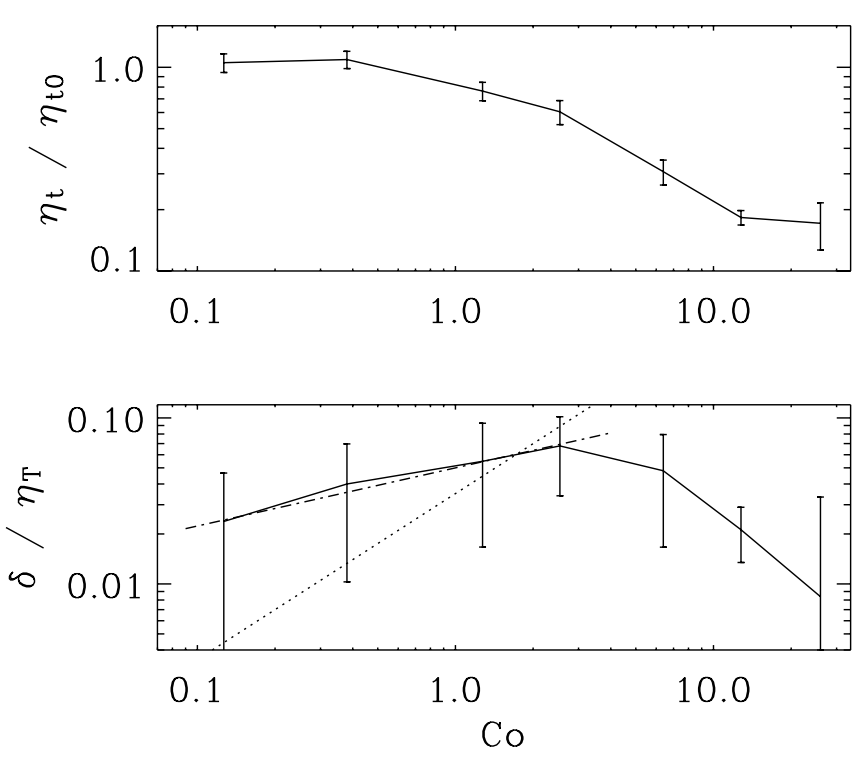

FIG. 1.-Dependence of the normalized $\eta_{t}$ and $\delta$ on Co for homogeneous turbulence with rotation for $\mathrm{Re}=1.3$ and $\operatorname{Re}_{M}=13$. The vertical lines indicate error bars. Note that there is a maximum of $\delta / \eta_{T}$ at $\mathrm{Co} \approx 3$. For $\mathrm{Co}<3$ the results for $\delta / \eta_{T}$ are best described by $\delta / \eta_{T} \approx 0.05 \mathrm{Co}^{0.35}$, given by the dash-dotted line, but also a linear dependence, $\delta / \eta_{T} \approx 0.035 \mathrm{Co}$, indicated by the dotted line, is compatible within error bars.

up to $256^{3}$ mesh points for the runs with the largest Reynolds numbers. As mentioned above, a computational domain of size $L^{3}$ is used, so the smallest finite wavenumber is $k_{1}=2 \pi / L$. As initial conditions for the hydrodynamic part we assume vanishing velocity, $\boldsymbol{U}=\mathbf{0}$, and uniform density equal to some value $\rho_{0}$. The initial condition in the test field calculations is $\boldsymbol{a}^{q}=\mathbf{0}$. Owing to the use of periodic boundary conditions, the total mass in the computational domain is conserved, and therefore, the mean density will be always equal to the initial value, $\langle\rho\rangle=\rho_{0}$, where the angle brackets denote a volume average.

In all investigations reported in this paper, only weakly compressible turbulence has been considered. The Mach number $u_{\text {rms }} / c_{s}$ did not exceed a value of the order of 0.1 .

\section{RESULTS FOR THE DIFFUSIVITY COEFFICIENTS}

As explained above, the test field procedure yields the coefficients $\eta_{i j}$ first as functions of $z$ and $t$. However, after averaging over sufficiently long time intervals, we expect to approach the results for homogeneous, isotropic, mirror-symmetric, and statistically steady background turbulence, in particular, coefficients $\eta_{i j}$, being independent of $z$ and $t$. We present here results for the $\eta_{i j}$ gained by averaging of the "raw" data first over $z$ and then over time. In this context, the effect of averaging over $z$ consists in a first reduction of the temporal fluctuations. This appears plausible in the picture in which the domain contains a finite number of turbulent "eddies" (Hoyng 1993). We may interpret them as different realizations of a specific eddy and, thus, the average over the $x, y$, and $z$ of a given domain as an average over the ensemble of these realizations. When accepting the principle that the ensemble average is equivalent to a time average we see that the effect of averaging the original $\eta_{i j}$ over $z$ is just equivalent to some temporal smoothing. After having averaged over $z$, time averages are then taken over a suitable stretch of the full time series where these averages are approximately steady. We use the time series further to calculate error bars as the maximum departure between these averages and the averages obtained from one of three equally long subsections of the full time series.
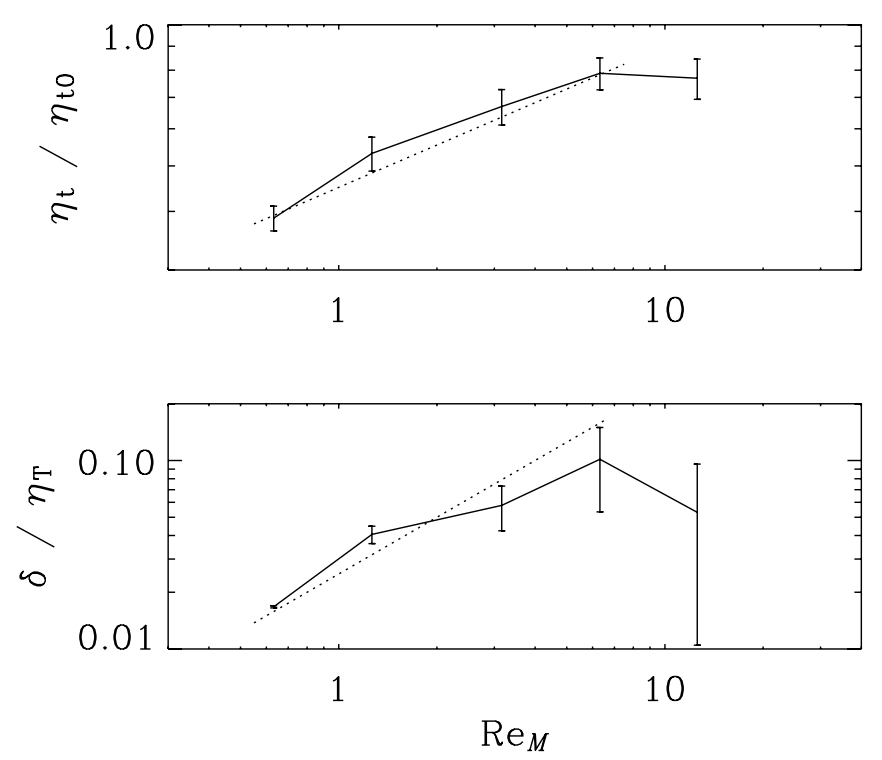

FIG. 2.-Dependence of the normalized $\eta_{t}$ and $\delta$ on $\operatorname{Re}_{M}$ for homogeneous turbulence with rotation for $\mathrm{Re}=1.3$ and $\mathrm{Co}=1.3$. The dotted lines show the powerlaw fits $\eta_{t} / \eta_{t 0}=0.45 \mathrm{Re}_{M}^{0.3}$ and $\delta / \eta_{T}=0.025 \mathrm{Re}_{M}$ which apply for $\operatorname{Re}_{M}<7$.

Important control parameters that are being varied include the hydrodynamic and magnetic Reynolds numbers, $\mathrm{Re}$ and $\mathrm{Re}_{M}$, as well as the magnetic Prandtl number $\operatorname{Pr}_{M}$, with

$$
\operatorname{Re}=u_{\mathrm{rms}} /\left(\nu k_{f}\right), \quad \operatorname{Re}_{M}=u_{\mathrm{rms}} /\left(\eta k_{f}\right), \quad \operatorname{Pr}_{M}=\nu / \eta
$$

In the case of rotation we define further the Coriolis number Co and in the case of shear we define the parameter Sh,

$$
\mathrm{Co}=2 \Omega /\left(u_{\mathrm{rms}} k_{f}\right), \quad \mathrm{Sh}=S /\left(u_{\mathrm{rms}} k_{f}\right) .
$$

We note that Co, like $\Omega$, is never negative. In all cases with shear presented below, $S$ and thus Sh are negative. For most of the calculations we use $k_{f} / k_{1}=5$, except in $\S 4.1$ where $k_{f} / k_{1}=10$. In both cases the range of forcing wavenumbers is $k_{f} \pm k_{1} / 2$.

\subsection{Effect of Rotation}

In the case of rotation $(\mathrm{Co} \neq 0)$, but without shear $(\mathrm{Sh}=0)$, the coefficients $\eta_{t}$ and $\delta$ are relevant. Figure 1 shows their dependence on the Coriolis number Co for fixed Reynolds numbers, $\mathrm{Re}=1.3$ and $\mathrm{Re}_{M}=13$. We see that $\eta_{t}$ shows a drastic decline when Co approaches and exceeds unity. This can be understood as a consequence of an evolving Taylor-Proudman state of the turbulent flow. Clearly, $\delta$ is positive. The ratio $\delta / \eta_{T}$ first increases with $\mathrm{Co}$, but it begins to decline when Co has exceeded a value of about 3 . In Figure 2 the dependence of $\eta_{t}$ and $\delta$ on $\operatorname{Re}_{M}$ is given for $\mathrm{Re}=16$ and $\mathrm{Co}=1.3$. As $\mathrm{Re}_{M}$ is increased, $\eta_{t}$ and $\delta$ increase for $\operatorname{Re}_{M}<10$.

According to the considerations in $\S 2.1$ we have to expect that the diagonal elements $\eta_{11}$ and $\eta_{22}$ of the magnetic diffusivity tensor coincide. Indeed, the observed values of $\epsilon$ (not shown) are only of the order of the errors.

Our results are consistent with those obtained in the framework of the second-order correlation approximation (see Appendix B). We take this consistency as a confirmation of the correctness of the test field method.

\subsection{Effect of Shear}

We now discuss the case of shear $(\mathrm{Sh} \neq 0)$ in the absence of rotation $(\mathrm{Co}=0)$. Figure 3 demonstrates that the value of $\eta_{t} / \eta_{t 0}$ 

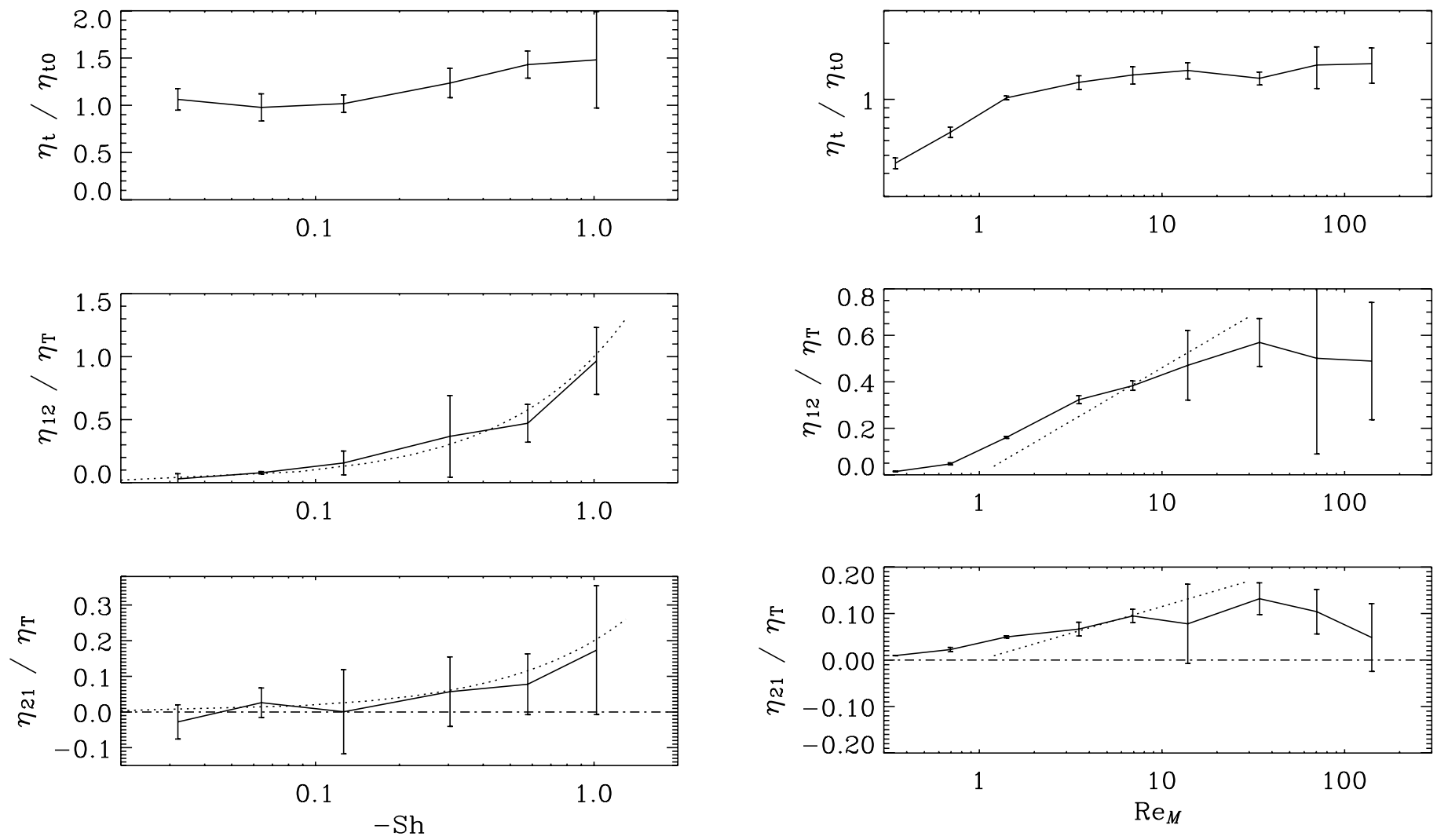

FIG. 3.-Dependence of $\eta_{t}$ (normalized by $\eta_{t 0}$ ) as well as $\eta_{12}$ and $\eta_{21}$ (both normalized by $\eta_{T}$ ) for homogeneous turbulence with shear on $\mathrm{Sh}$ for $\mathrm{Re}=1.4$ and $\mathrm{Re}_{M}=14$. The dotted lines represent linear dependences on Sh.

clearly exceeds unity for not too small values of $|\mathrm{Sh}|$, that is, shear leads to a slight enhancement of the turbulent magnetic diffusivity. At the same time, for negative values of Sh, both $\eta_{12}$ and $\eta_{21}$ attain finite positive values. In Figures 4 and 5 these quantities are shown as functions of $\mathrm{Re}_{M}$, with $\mathrm{Re}=1.4$ and $\mathrm{Sh}=-0.6$, or $\operatorname{Pr}_{M}=20$, respectively.

We recall the dynamo condition from equation (29). Since in all our simulations $S$ is negative, a dynamo would be possible for negative $\eta_{21}$ only. In Figure 5, with $\operatorname{Pr}_{M}=20$, we see indeed negative $\eta_{21}$ for high $\mathrm{Re}_{M}$. Considering the large error bars, however, we may hardly conclude that a dynamo is really possible. In general, the errors could be reduced by extending the time series. However, for large $\mathrm{Re}_{M}$ small-scale dynamo action occurs that introduces additional fluctuations whose amplitude increases exponentially with time, and so we have to stop the calculation. One remedy might be to reset $\boldsymbol{b}^{q}$ in regular time intervals, but this has not been done yet.

As the considerations of $\S 2.1$ show, there is no general reason for an equality of the two diagonal elements $\eta_{11}$ and $\eta_{22}$ of the diffusivity tensor, that is, $\epsilon$ does not need to be equal to zero. As shown in Figure 6, for $\mathrm{Re}_{M}$ of order 10 and above, $\epsilon$ may deviate from zero, but its value is of the order of the error. Again, our numerical results are in agreement with results obtained in the second-order correlation approximation (see Appendix B).

\section{COMPARISON WITH DIRECT SIMULATIONS AND SIMPLIFIED MODELS}

\subsection{Large-scale Fields in Simulations with Shear}

We report now on calculations with the original induction equation (15), instead of the test field equation (20), together with the hydrodynamic equations (9) and (10). In the momen-

FIG. 4.-Dependences of $\eta_{t}$ (normalized by $\eta_{t 0}$ ), as well as $\eta_{12}$ and $\eta_{21}$ (both normalized by $\eta_{T}$ ) for homogeneous turbulence with shear on $\operatorname{Re}_{M}$ for $\mathrm{Re}=1.4$ and $\mathrm{Sh}=-0.6$. The dotted lines correspond to $\eta_{12} / \eta_{T}=0.2 \ln \operatorname{Re}_{M}$ and $\eta_{21} / \eta_{T}=$ $0.05 \ln \operatorname{Re}_{M}$ and illustrate that $\eta_{12} / \eta_{T}$ and $\eta_{21} / \eta_{T}$ vary only weakly with $\operatorname{Re}_{M}$.

tum equation (9), however, the Lorentz force was restored, thus providing a nonlinear feedback of the magnetic field. In all cases we used $\mathrm{Sh}=-0.15$ and $k_{f} / k_{1}=10$. The results are shown in Figures 7 and 8 for two different combinations of $\operatorname{Re}_{M}$ and $\operatorname{Pr}_{M}$. In both cases there is an initial phase where the mean field grows exponentially. Mean fields with a particularly prominent $\bar{B}_{y}$ component occur. The $\bar{B}_{x}$ component seems to be in antiphase with $\bar{B}_{y}$, as expected for negative shear, but this component is much more noisy. Furthermore, for $\operatorname{Pr}_{M}=7$ and $\mathrm{Re}_{M}=130$ (Fig. 7) there are episodes where $\bar{B}_{y}$ fades away and later reemerges, but possibly with the opposite orientation. Similar results (not shown here) have also been obtained for smaller values of $\operatorname{Re}_{M}$. In the case with $\operatorname{Pr}_{M}=20$ and $\operatorname{Re}_{M}=200$ (Fig. 8), however, $\bar{B}_{y}$ keeps the same orientation throughout the run.

\subsection{Magnitude and Effect of Fluctuations}

In $\S 3.2$ we have seen that the sign of $\eta_{21}$ is not suitable for enabling a shear-current dynamo except perhaps for high values of $\mathrm{Re}_{M}$. On the other hand, as demonstrated in $\S 4.1$, large-scale magnetic fields are being generated. Explaining this in terms of the shear-current effect is very questionable. Therefore, we ask now whether an incoherent alpha-shear dynamo (Vishniac \& Brandenburg 1997) might play a role. Another explanation would be an incoherent shear-current dynamo that we discuss below in $\S 4.3$.

The possibility that a random $\alpha$ with zero mean can produce magnetic fields was first discussed by Kraichnan (1976) and Moffatt (1978). In the presence of shear, strong large-scale fields can be generated (Vishniac \& Brandenburg 1997; Sokolov 1997; Silant'ev 2000; Fedotov et al. 2006; Proctor 2007). Consider an incoherent alpha-shear dynamo with a scalar $\alpha$ fluctuating around 

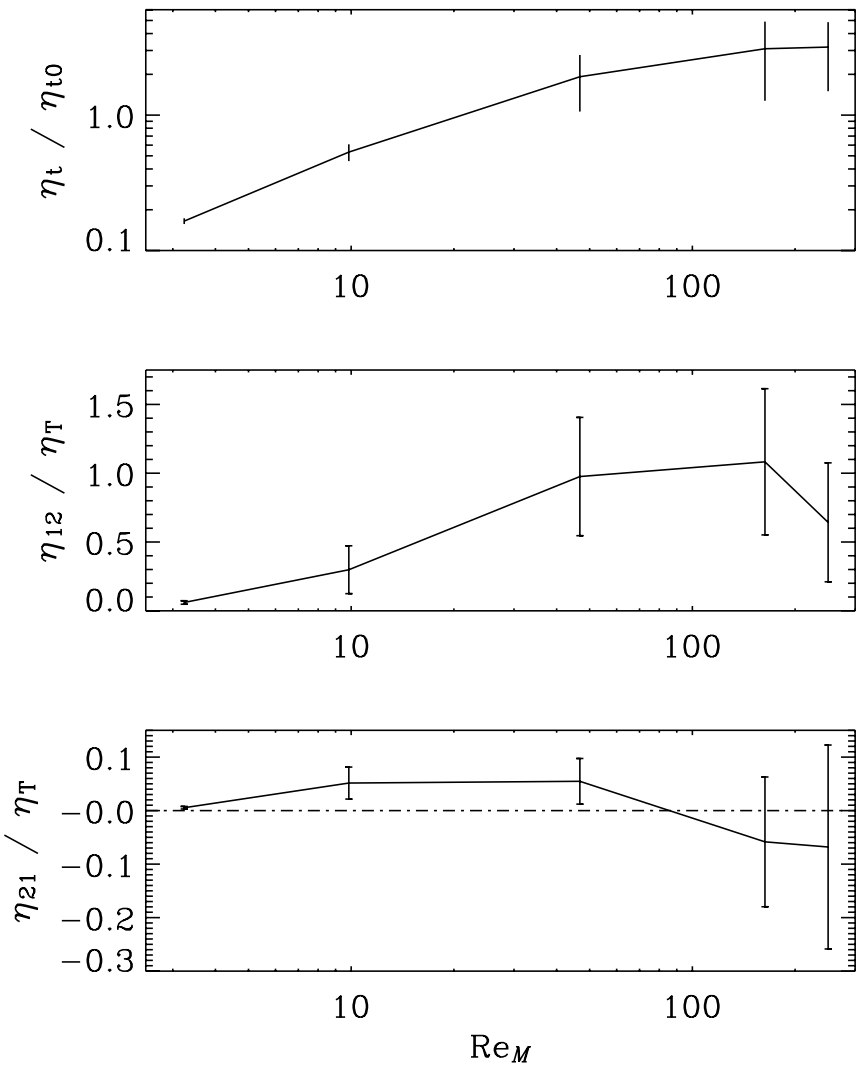

FIG. 5.- Same as Fig. 4, but for a fixed magnetic Prandtl number, $\operatorname{Pr}_{M}=20$. Here, $\mathrm{Re}=\mathrm{Re}_{M} / 20$ is not constant. Because $u_{\text {rms }}$ increases with increasing $\operatorname{Re}_{M}$, Sh is also not constant and varies between -2.5 (for $\operatorname{Re}=0.16$ ) and -0.3 (for $\operatorname{Re}=13$ ). Note that $\eta_{21}$ turns negative at about $\operatorname{Re}_{M}=100$. However, the errors are larger than the mean.

zero. In the limit $k \ll k_{f}$ and if $|\alpha| k \ll|S|$, the condition for mean fields growing on the average exponentially reads

$$
D_{\alpha S}=\alpha_{\mathrm{rms}}|S| /\left(\eta_{T}^{2} k^{3}\right)>D_{\alpha S}^{\mathrm{crit}},
$$

where $D_{\alpha S}^{\text {crit }} \approx 2.3$ for a white-noise $\alpha$ effect (see Appendix C).

In a finite domain all mean-field coefficients show fluctuations, and so $\alpha_{i j}$ must fluctuate about zero. We may extend the test field procedure for the determination of the $\eta_{i j}$ such that it provides us the $\alpha_{i j}$ too. When starting from equation (2) instead of equation (8) and using again the four test fields $\overline{\boldsymbol{B}}^{q c}$ and $\overline{\boldsymbol{B}}^{q S}, q=1,2$, we find

$$
\begin{aligned}
& \alpha_{i 1}=B^{-1}\left(\overline{\mathcal{E}}_{i}^{1 c} \cos k z+\overline{\mathcal{E}}_{i}^{1 s} \sin k z\right), \\
& \alpha_{i 2}=B^{-1}\left(\overline{\mathcal{E}}_{i}^{2 c} \cos k z+\overline{\mathcal{E}}_{i}^{2 s} \sin k z\right), \quad i=1,2,
\end{aligned}
$$
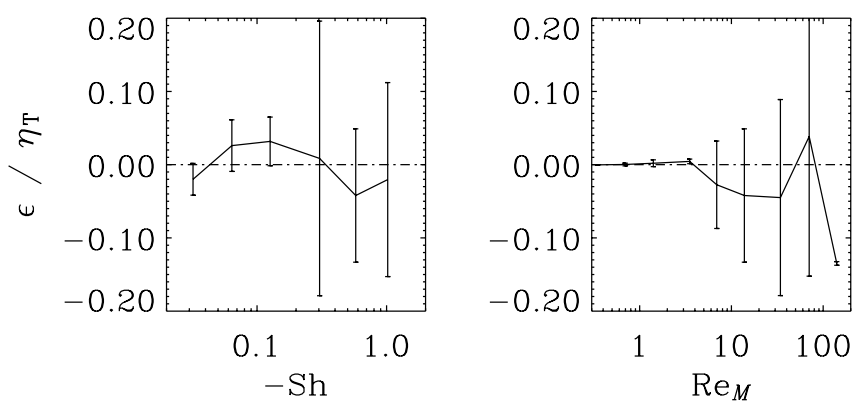

Fig. 6.-Dependences of $\epsilon$ (normalized by $\eta_{T}$ ) on Sh (left $)$ and on $\operatorname{Re}_{M}(r i g h t)$ for the same runs as in Figs. 3 and 4, respectively.
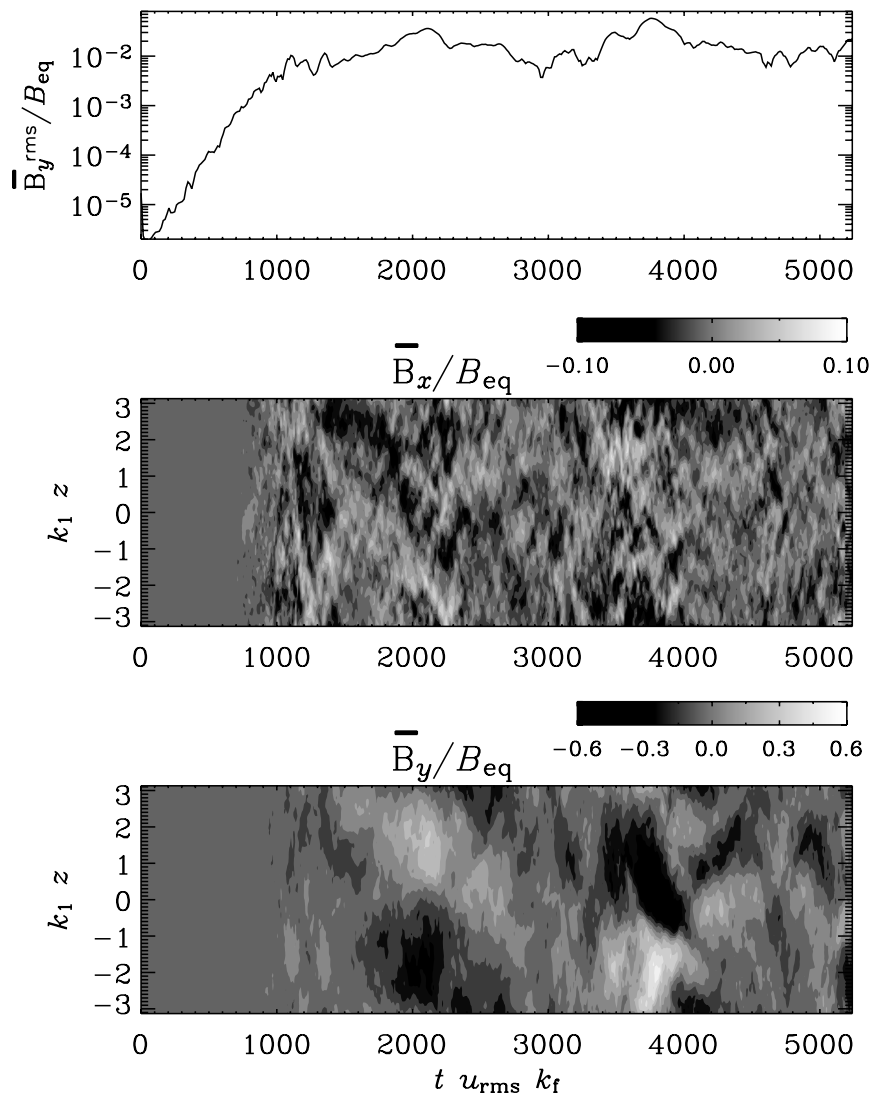

FIG. 7.- Time dependence of the rms value (with respect to $z$ ) of $\bar{B}_{y}$ (top) and spacetime diagrams $\bar{B}_{x}(z, t)$ and $\bar{B}_{y}(z, t)$ [all in units of $B_{\text {eq }}$, where $B_{\text {eq }}=$ $\left.\left(\mu_{0}\left\langle\rho u^{2}\right\rangle\right)^{1 / 2}\right]$ from a direct simulation with $\operatorname{Re}_{M}=130, \operatorname{Pr}_{M}=7, k_{f} / k_{1}=10$, and $\mathrm{Sh} \approx-0.15$. The top panel demonstrates the initial exponential growth of the mean field (the growth rate is $0.009 u_{\mathrm{rms}} k_{f}$ ). The other panels show episodes of large scales in $z$ especially in the $\bar{B}_{y}$ component. [See the electronic edition of the Journal for a color version of this figure.]

together with the relations from equation (23) for $\eta_{i 1}$ and $\eta_{i 2}$ (see also Brandenburg 2005b).

In contrast to the considerations in $\S 3$ we consider now the mean-field coefficients, as obtained from the test field calculations, after averaging over $z$, but not over $t$. Then the $\alpha_{i j}$ consist of fluctuations around a zero mean, that is, we have an incoherent $\alpha$ effect. (Without the averaging over $z$, the fluctuations would be even bigger.) In the case of fluctuations of $\eta_{i j}$ we speak analogously about an incoherent shear-current effect.

We have calculated the rms values of the temporal fluctuations of the $\alpha_{i j}$ and $\eta_{i j}$ which are denoted by $\alpha_{i j}^{\text {rms }}$ and $\eta_{i j}^{\text {rms }}$, respectively. We have also taken the averages over all four components of $\alpha_{i j}^{\mathrm{rms}}$ and over the two diagonal components of $\eta_{i j}^{\mathrm{rms}}$ and denoted them by $\alpha^{\mathrm{rms}}$ and $\eta_{t}^{\mathrm{rms}}$, respectively. Figure 9 shows these quantities along with $\eta_{12}^{\text {rms }}$ and $\eta_{21}^{\text {rms }}$ for a small Reynolds number and moderate shear as functions of $\operatorname{Re}_{M}$. They are all mildly growing.

In Figure 10 we show that the probability density functions of $\alpha_{i j}$ and $\eta_{i j}$ for a run with $\operatorname{Re}_{M}=14, \mathrm{Re}=1.4$, and $\mathrm{Sh}=-0.6$ are approximately Gaussian. In order to improve the statistics we have, in addition, averaged the results for all four components of $\alpha_{i j}$. The result is similar to those for the individual components. The diagonal components of $\eta_{i j}$ are distributed around finite averages, and $\eta_{21}$ is distributed around a positive, but small value.

\subsection{An Incoherent Shear-Current Dynamo?}

Yousef et al. (2007) have reported large-scale dynamo action at low Reynolds numbers $\left(\mathrm{Re}=\mathrm{Re}_{M}=5\right)$ for weak shear 

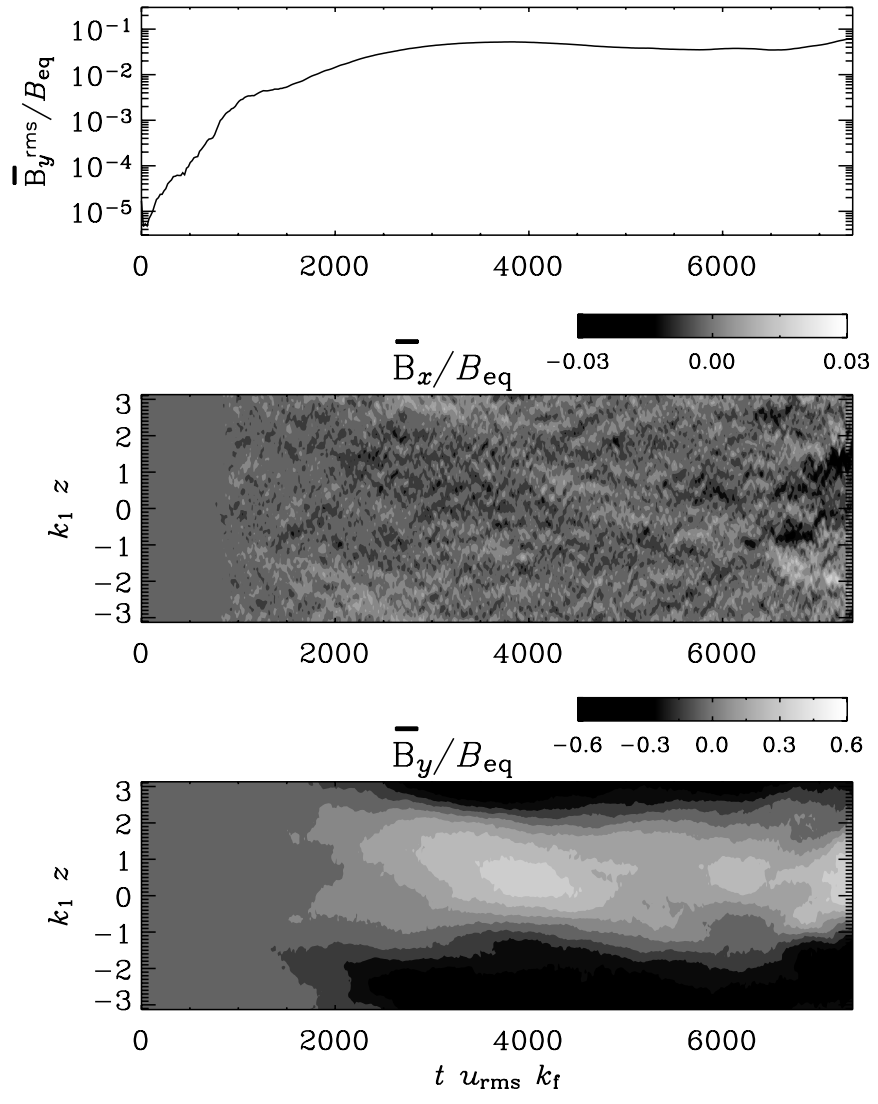

FIG. 8. - Same as Fig. 7, but for $\operatorname{Re}_{M}=200$ and $\operatorname{Pr}_{M}=20$. Initially, the field grows exponentially at a rate $0.012 u_{\mathrm{rms}} k_{f}$. Note that the mean field is nearly steady. [See the electronic edition of the Journal for a color version of this figure.]

[Sh $\left.<(3 \pi)^{-1}\right]$ in tall boxes so that the smallest wavenumber in the $z$-direction, $k_{1 z}$, can be up to 128 times smaller than those in the other two directions. They discuss in more detail the case where it is 16 times smaller, i.e., $k_{1 z}=k_{1} / 16$. Using small Reynolds numbers has the advantage that small-scale dynamo action is then impossible.

We have analyzed similar cases $\left(\mathrm{Re}=5\right.$ with $\operatorname{Pr}_{M}=1$ and $0.01<-$ Sh $<0.3$ ) using, however, cubic domains of size $L^{3}$, so $k_{1 z}=k_{1}$, and a forcing with $k_{f} / k_{1}$ equal to 5 instead of 3 . It turns out that the value of the crucial coefficient $\eta_{21}$ fluctuates around zero. This is also plausible from Figure 3 (even if it

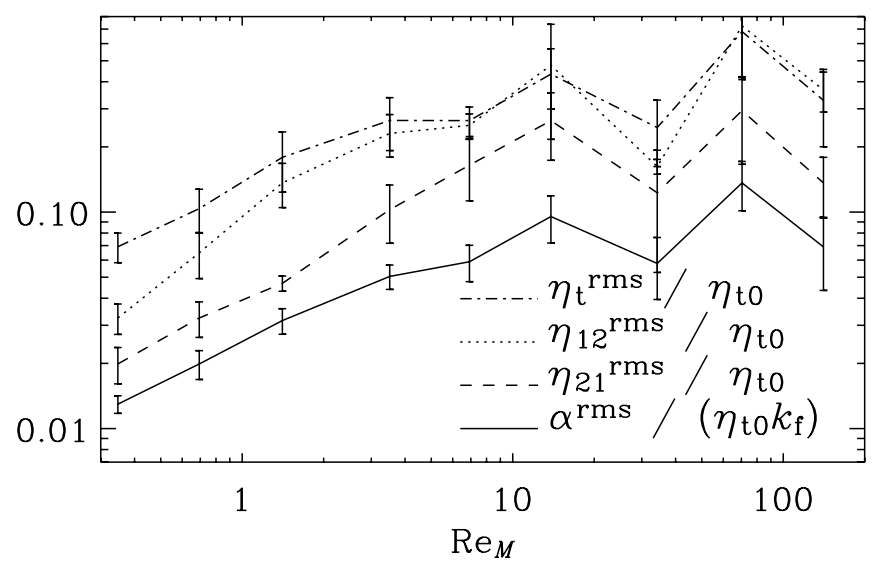

Fig. 9.-Dependences of the rms values of the temporal fluctuations $\alpha^{\text {rms }}$ (normalized by $\eta_{t 0} k_{f}$ ), $\eta_{t}^{\text {rms }}, \eta_{21}^{\mathrm{rms}}$, and $\eta_{12}^{\mathrm{rms}}$ (normalized by $\eta_{t 0}$ ) on $\mathrm{Re}_{M}$ for $\mathrm{Re}=$ 1.4 and $\mathrm{Sh}=-0.6$.
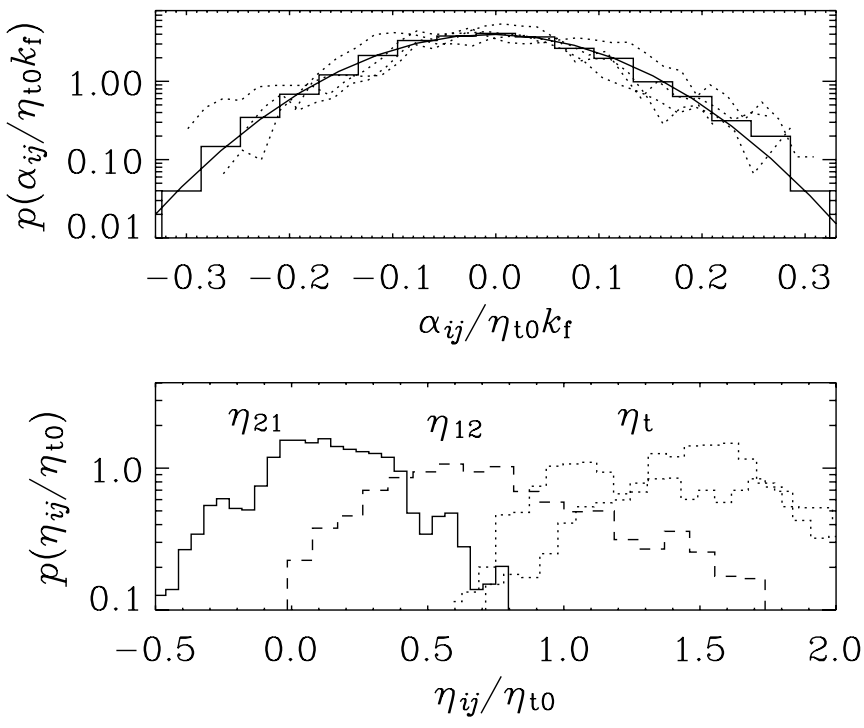

FIG. 10.-Probability density functions (PDFs) of $\alpha_{i j}$ (top) and $\eta_{i j}$ (bottom) for $\mathrm{Re}_{M}=14, \mathrm{Re}=1.4$, and $\mathrm{Sh}=-0.6$. The PDFs for the different components of $\alpha_{i j}$ (dotted lines) are close together; their average is given by the solid staircase line and compared with a Gaussian fit. The PDF of $\eta_{21}$ (solid line) is around zero, while those of $\eta_{12}, \eta_{11}$, and $\eta_{22}$ are not. (The latter two are simply denoted by $\eta_{t}$.)

does not apply to $\operatorname{Pr}_{M}=1$ ). We must therefore conclude that the (coherent) shear-current effect cannot explain the generation of the mean magnetic field found by Yousef et al. (2007).

With respect to the incoherent effects, it can be seen from Figure 11 that the values of $\alpha^{\mathrm{rms}}$ and $\eta_{21}^{\mathrm{rms}}$ are more or less the same for different $\operatorname{Pr}_{M}, \operatorname{Re}_{M}$, and Sh. Let us consider the instantaneous values of the growth rate $\lambda$, as calculated from equation (28) with the fluctuating $\eta_{i j}$. If $k=k_{1}$, we get always negative $\lambda$. However, for the smaller value $k=k_{1} / 16$, appropriate for the model of Yousef et al. (2007), it is possible to have large positive $\lambda$ during extended periods of time. Although $\overline{\boldsymbol{B}}$ can be amplified during those episodes, it must decay during episodes with the reversed sign of $\eta_{21}$, and it is not certain from this competition whether a dynamo powered by the incoherent shear-current effect may result.

So far we have ignored the possibility of an incoherent alphashear dynamo that must work at the same time. In order to assess the relative importance of the two incoherent effects we have considered a simple model with random $\boldsymbol{\alpha}$ and $\boldsymbol{\eta}$ tensors that are delta-correlated in time. (Delta-correlated noise is the simplest model; a more realistic case would be to assume colored noise

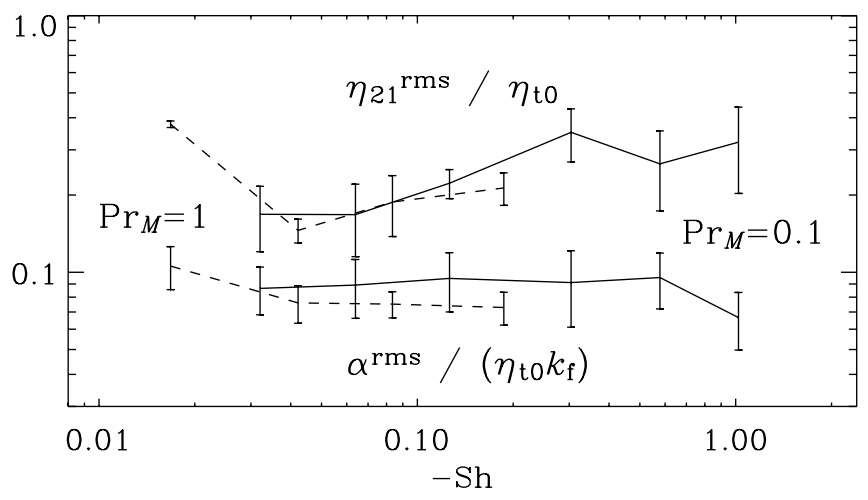

FIG. 11.-Dependence of the rms values of the temporal fluctuations $\alpha^{\mathrm{rms}}$ (normalized by $\eta_{t 0} k_{f}$ ) and $\eta_{21}^{\mathrm{rms}}$ (normalized by $\eta_{t 0}$ ) on Sh for models with $\operatorname{Pr}_{M}=1$ and $\mathrm{Re}=5$ (dashed lines) compared with the models shown in Fig. 3 with $\operatorname{Pr}_{M}=0.1$ and $\operatorname{Re}_{M}=14$ (solid lines). 


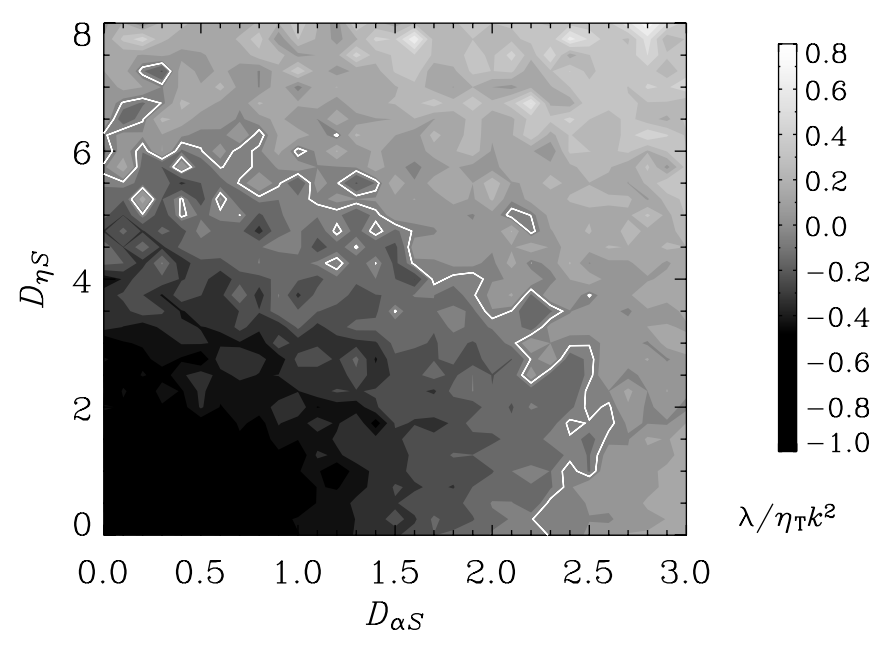

FIG. 12.-Contour plot showing the normalized growth rate as a function of the dynamo numbers for the incoherent alpha-shear and shear-current dynamos from a numerical solution of the model described in $\S 4.3$. The zero line is given in white. [See the electronic edition of the Journal for a color version of this figure.]

with a finite correlation time.) The model is explained in Appendix C. Under the assumptions $\left|\alpha_{11}\right| \ll|S| / k$ and $\left|\alpha_{12}\right|,\left|\alpha_{21}\right| \ll$ $\eta_{T} k$, as well as $\left|\eta_{12}\right| \ll|S| / k^{2}$ and $|\epsilon| \ll\left|\eta_{T}\right|$, its governing parameters are the two dynamo numbers for the incoherent effects,

$$
D_{\eta S}=C_{\eta} C_{S}, \quad D_{\alpha S}=C_{\alpha} C_{S}
$$

which can be expressed in terms of the three quantities

$$
C_{S}=\frac{|S|}{\eta_{T} k^{2}}, \quad C_{\alpha}=\frac{\alpha_{22}^{\mathrm{rms}}}{\eta_{T} k}, \quad C_{\eta}=\frac{\eta_{21}^{\mathrm{rms}}}{\eta_{T}} .
$$

In Figure 12 we give a contour plot of the normalized growth rate as a function of the two dynamo numbers, $D_{\alpha S}$ and $D_{\eta S}$. For small values of $D_{\eta S}$, the incoherent shear-current effect has a slightly adverse effect on the dynamo, but for larger values, it lowers the critical value of $D_{\alpha S}$ significantly. For $D_{\alpha S}=0$, even a purely incoherent shear-current dynamo is possible if $D_{\eta S}>6.5$.

In interpreting simulations, we focus on domains whose smallest finite wavenumber in the $z$-direction is $k_{1 z}$. In view of the already discussed dynamo found by Yousef et al. (2007) we employ the data for $\alpha_{i j}^{\mathrm{rms}}$ and $\eta_{i j}^{\mathrm{rms}}$ originating from our aforementioned calculations of similar cases to derive growth rates with the help of Figure 12. With $k=k_{1 z}=k_{1} / 16$ we find first $C_{S} \approx 40$, and from Figures 5 and 11 , we have $C_{\eta} \approx 0.1$ and $C_{\alpha} \approx 0.1$ (using $k_{f} / k_{1}=3$ ), so that $D_{\eta S} \approx 4$ and $D_{\alpha S} \approx 4$. This suggests that the incoherent shear-current dynamo is subcritical, while the incoherent alpha-shear dynamo is supercritical. Therefore, an incoherent alpha-shear dynamo seems to be a plausible explanation. This explanation is further supported by our finding that for constant rms values the growth rate is in good approximation a linear function of $S$-just as observed by Yousef and coworkers.

An explanation in terms of the incoherent $\alpha$ effect is also suited for the nonhelical dynamo of Brandenburg (2005a), where $C_{S} \approx 25, C_{\eta} \approx 0.15$, and $C_{\alpha} \approx 0.2$ for the appropriate value of the magnetic Reynolds number, $\operatorname{Re}_{M}=80$, so that $D_{\eta S} \approx 4$ and $D_{\alpha S} \approx 5$. For this model we used $k_{f} / k_{1}=5$, although the shear profile is here more complicated.

Finally, let us return to the cases of dynamo action considered in Figures 7 and 8. If we assume that the negative values of $\eta_{21}$ seen in Figure 5 for $\operatorname{Re}_{M}>100$ are real, we have to ask for the relative importance of the regular (coherent) shear-current effect and the two incoherent effects. For both dynamo cases in Figures 7 and 8 , the values of $C_{S}$ are $\sim 30$ (but uncertain because the dynamo-generated field quenches the value of $\eta_{t}$ ), so the three dynamo numbers would be $D_{\eta S} \approx 1.5$ for the regular shearcurrent effect, $D_{\eta S}=1.5-3$ for the incoherent one and $D_{\alpha S}=$ 3-6, respectively, where we have used $C_{\eta}=0.05-0.1$ and $C_{\alpha}=$ $0.1-0.2$ (cf. Figs. 5, 9, and 11). The corresponding critical values are $1,6.5$, and 2.3 , respectively. Hence, with respect to the regular shear-current dynamo this case is only slightly supercritical, but subcritical with respect to the incoherent shear-current effect and supercritical with respect to the incoherent alpha-shear dynamo. By inspection of the values of the growth rate it is possible to infer safely that this situation is dominated by the incoherent $\alpha$ effect. The incoherent shear-current effect has a weakly adverse influence, whereas its regular counterpart clearly supports dynamo action. The values of $D_{\alpha S}$ and $D_{\eta S}$ could be somewhat smaller if one takes into account that the level of fluctuations is smaller for $k_{f} / k_{1 z}=10$ instead of 5 .

\section{CONCLUSIONS}

The present work has demonstrated that the test field method provides a robust means of determining all components of the turbulent magnetic diffusivity tensor that are relevant for mean fields depending only on $z$ and $t$. Both rotating and weakly shearing turbulence are studied. In either case, the diagonal components of the turbulent diffusivity tensor are about equal to each other. Shear slightly enhances the turbulent magnetic diffusivity, while rotation quenches it. In the presence of rotation, the $\Omega \times \bar{J}$ effect occurs, which is described by the off-diagonal components of the turbulent magnetic diffusivity tensor. Shear leads to the shear-current effect, again described by off-diagonal components of this tensor. In both cases, the results are consistent with those found in the framework of the second-order correlation approximation.

The possibility of the so-called shear-current dynamo has been scrutinized. It depends crucially on the sign of the component $\eta_{21}$ of the magnetic diffusivity tensor. It turns out that, within the ranges of parameters considered, its sign is in general not suited for driving a dynamo based on this effect, with a possible exception at large magnetic Reynolds numbers. In this way, the analytic results found in the second-order correlation approximation for incompressible fluids (Rüdiger \& Kitchatinov 2006; Rädler \& Stepanov 2006) are confirmed and generalized.

Direct numerical simulations are presented which exhibit growing mean magnetic fields in shear flow turbulence. An interpretation as a (coherent) shear-current dynamo is hardly possible. Instead, it is argued that it can be explained by an incoherent alpha-shear dynamo. The incoherent shear-current effect has also been determined, but it is found to be less important.

A great deal of the work presented here was done during stays of K.-H. R. and M. R. at NORDITA. They are grateful for the hospitality. A. B. thanks the Kavli Institute for Theoretical Physics for hospitality. This research was supported in part by the National Science Foundation under grant PHY 05-51164. P. J. K. acknowledges financial support from the Helsingin Sanomat Foundation. We thank the Centers for Scientific Computing in Denmark (DCSC), Finland (CSC), and Sweden (PDC) for the allocation of computing resources. 


\section{APPENDIX A}

\section{CONCERNING $\alpha_{i j}=0$}

In the case of rotation the tensorial structure of $\alpha_{i j}$ must agree with that of $\eta_{i j}$ given in equation (3), that is,

$$
\alpha_{i j}=\alpha_{0} \delta_{i j}+\alpha_{1} \epsilon_{i j k} \hat{\Omega}_{k}+\alpha_{2} \hat{\Omega}_{i} \hat{\Omega}_{j}
$$

Since $\alpha_{i j}$ is a pseudo-tensor and $\Omega$ an axial vector, the coefficients $\alpha_{0}, \alpha_{1}$, and $\alpha_{2}$ must be pseudo-scalars. Under our assumptions, however, no pseudo-scalars can be constructed. So we have to conclude that $\alpha_{i j}=0$.

In the case of shear we may argue analogously. Referring to equation (5) and the subsequent explanations we have then

$$
\alpha_{i j}=\alpha_{0} \delta_{i j}+\alpha_{1} g_{i} g_{j}+\alpha_{2} h_{i} h_{j}+\alpha_{3} g_{i} h_{j}+\alpha_{4} g_{j} h_{i}
$$

with $\alpha_{0}, \alpha_{1}, \ldots, \alpha_{4}$ being pseudo-scalars. Again, it is impossible to construct pseudo-scalars. Thus, we have again $\alpha_{i j}=0$. Of course, the situation would be different if the shear provided (large-scale) kinetic helicity, as then the pseudoscalar $\boldsymbol{U}^{S} \cdot\left(\nabla \times \boldsymbol{U}^{S}\right)$ would be available.

\section{APPENDIX B}

\section{COMPARISON WITH RESULTS OF THE SECOND-ORDER CORRELATION APPROXIMATION}

In a paper by Rädler \& Stepanov (2006), the mean electromotive force has been calculated in the second-order correlation approximation for generally inhomogeneous turbulence in an incompressible rotating fluid showing a position-dependent mean motion. In this context, the second-order correlation approximation was understood as the neglect of higher order terms in the induction equation as well as in the momentum balance. Both the Coriolis force and derivatives of the mean velocity were assumed to be small enough so that the mean electromotive force is linear in the angular velocity $\boldsymbol{\Omega}$ and the gradient tensor of $\overline{\boldsymbol{U}}$. Detailed results were obtained for a special correlation function of the background turbulence.

Let us apply the results to the situations considered in the present paper. In the case of rotation without shear we obtain

$$
\frac{\delta}{\eta_{t}}=\frac{1}{4} \sqrt{\frac{\pi}{2}} \operatorname{CoRe}_{M}\left(\lambda_{c} k_{f}\right)^{2} \sqrt{q} \delta^{0}\left(q, \operatorname{Pr}_{M}\right)
$$

with Co, $\operatorname{Re}_{M}$, and $\operatorname{Pr}_{M}$ as defined above, $q=\lambda_{c}^{2} / \eta \tau_{c}$, and $\lambda_{c}$ and $\tau_{c}$ being correlation length and time, respectively. When introducing the Strouhal number St $=u_{\mathrm{rms}} k_{f} \tau_{c}$, we have $q=\mathrm{Re}_{M} / \mathrm{St}$. It seems plausible to assume that $\lambda_{c} k_{f} \approx 2 \pi$. The function $\delta^{0}$ can be calculated according to $\delta^{0}=\left[\delta^{0(\Omega)}\left(q, \operatorname{Pr}_{M}\right)+\kappa^{0(\Omega)}\left(q, \operatorname{Pr}_{M}\right)\right] / 2 \beta^{0(0)}(q)$ from the functions $\delta^{0(\Omega)}, \kappa^{0(\Omega)}$, and $\beta^{0(0)}$ defined and plotted in Rädler \& Stepanov (2006). It turns out that $\delta^{0}$ is never negative and approaches unity if $\operatorname{Pr}_{M}=1$ and $q \rightarrow 0$. Of course, we have $\delta / \eta_{T}=$ $\left(\delta / \eta_{t}\right)\left(\eta_{t} / \eta_{T}\right)$. The factor $\eta_{t} / \eta_{T}$ depends on $\operatorname{Re}_{M}, \lambda_{c} k_{f}$, and $\beta^{0(0)}(q)$. It satisfies $0 \leq \eta_{t} / \eta_{T}<1$ and approaches unity as $\operatorname{Re}_{M} \rightarrow \infty$.

Clearly, equation (B1) and the results reported in $\S 3.1$ agree in the sign of $\delta$. Although these results do not really confirm the linearity of $\delta$ in Co, which is suggested by equation (B1), they are not in conflict with that (see Fig. 1). A further comparison of results is difficult because of, e.g., the not exactly known value of $\lambda_{c}$ and the errors of the data presented above.

Proceeding to the case of shear without rotation we note first that, due to the aforementioned assumption on the linearity in the mean velocity gradient, that is, in $S$, both $\kappa_{11}$ and $\kappa_{22}$ are equal to zero. Furthermore, we have

$$
\frac{\eta_{12}}{\eta_{t}}=-\frac{3}{5} \operatorname{Sh} \operatorname{Re}_{M}\left(\lambda_{c} k_{f}\right)^{2} \eta_{12}^{0}\left(q, \operatorname{Pr}_{M}\right), \quad \frac{\eta_{21}}{\eta_{t}}=-\frac{3}{5} \operatorname{Sh} \operatorname{Re}_{M}\left(\lambda_{c} k_{f}\right)^{2} \eta_{21}^{0}\left(q, \operatorname{Pr}_{M}\right)
$$

Here $\eta_{12}^{0}=\frac{1}{2}\left[\eta^{0(D)}\left(q, \operatorname{Pr}_{M}\right)+\eta^{0(W)}\left(q, \operatorname{Pr}_{M}\right)\right]$ and $\eta_{21}^{0}=\frac{1}{2}\left[\eta^{0(D)}\left(q, \operatorname{Pr}_{M}\right)-\eta^{0(W)}\left(q, \operatorname{Pr}_{M}\right)\right]$, where $\eta^{0(D)}=\left[13 \kappa^{0(D)}\left(q, \operatorname{Pr}_{M}\right)-\right.$ $\left.7 \beta^{0(D)}\left(q, \operatorname{Pr}_{M}\right)\right] / 6 \beta^{0(0)}(q)$ and $\eta^{0(W)}=\left[5 \delta^{0(W)}\left(q, \operatorname{Pr}_{M}\right)+\kappa^{0(D)}\left(q, \operatorname{Pr}_{M}\right)\right] / 6 \beta^{0(0)}(q)$, with the functions $\kappa^{0(D)}, \beta^{0(D)}, \delta^{0(W)}, \kappa^{0(W)}$, and $\beta^{0(0)}$ of Rädler \& Stepanov (2006). The quantities $\eta_{12}^{0}$ and $\eta_{21}^{0}$ approach unity and zero, respectively, if $\operatorname{Pr}_{M}=1$ and $q \rightarrow 0$. We note that $-\eta_{12} / S$ and $\eta_{21} / S$ coincide with the quantities $\delta^{\prime}$ and $\delta$ introduced in Appendix D of Rädler \& Stepanov (2006), respectively. It has been shown there that this $\delta$ (different from that considered above) cannot take negative values. This applies then to $\eta_{21}$ too.

Being aware that the second-order approximation applies only for $\mathrm{Re}_{M}$ that is not too large, we may state that equation (B2) and the numerical results reported in $\S 3.2$ agree in the sign of $\eta_{21}$. The possible deviation in Figure 5 is outside the validity range of this approximation. The linearity of $\eta_{12}$ and $\eta_{21}$ in Sh indicated in equation (B2) is well confirmed by the numerical results (see Fig. 3). Again, further comparison of the results is, for the reasons mentioned above, rather difficult, but no striking disagreement has been found.

\section{APPENDIX C}

\section{INCOHERENT ALPHA-SHEAR AND SHEAR-CURRENT DYNAMOS}

We calculate numerically solutions of the dynamo equation with incoherent $\alpha$ and shear-current effects in unbounded space. It reads

$$
\frac{\mathcal{D} \overline{\boldsymbol{A}}}{\mathcal{D} t}=-S \bar{A}_{y} \hat{\boldsymbol{x}}+\overline{\mathcal{E}}-\eta \overline{\boldsymbol{J}}
$$



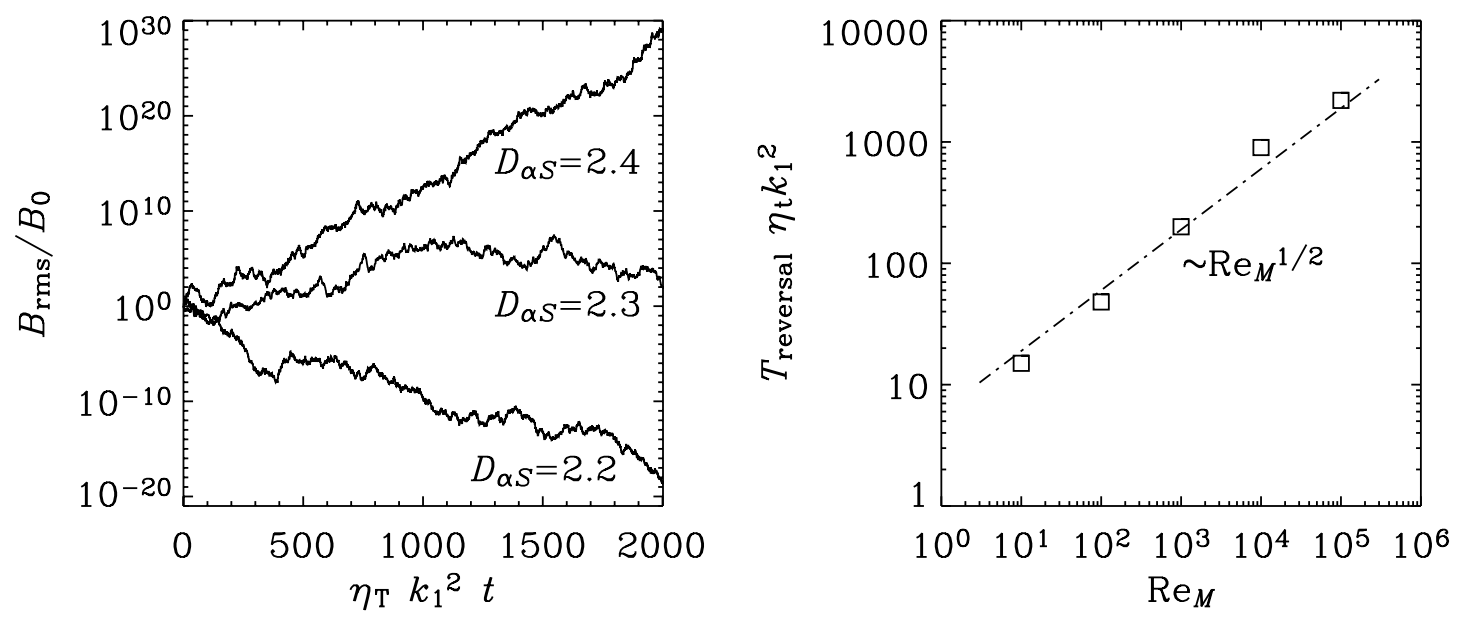

FIG. 13.- Results for an incoherent alpha-shear dynamo $\left(D_{\eta S}=0\right)$. Left: Temporal evolution curves of $B_{\text {rms }}$ showing that the critical value of $D_{\alpha S}$ is around 2.3 .

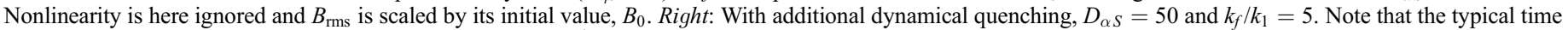
between reversals increases with $\operatorname{Re}_{M}$ approximately like $\operatorname{Re}_{M}^{1 / 2}$.

where

$$
\overline{\mathcal{E}}_{i}=\alpha_{i j}(t) \bar{B}_{j}-\eta_{i j}(t) \bar{J}_{j}
$$

The $\boldsymbol{\alpha}$ and $\boldsymbol{\eta}$ tensors are delta-correlated in time with

$$
\begin{gathered}
\left\langle\alpha_{i j}(t) \alpha_{i j}\left(t^{\prime}\right)\right\rangle=\left(\alpha_{i j}^{\mathrm{rms}}\right)^{2} \delta\left(t-t^{\prime}\right), \quad\left\langle\alpha_{i j}\right\rangle=0, \\
\left\langle\eta_{i j}(t) \eta_{i j}\left(t^{\prime}\right)\right\rangle-\left\langle\eta_{i j}\right\rangle^{2}=\left(\eta_{i j}^{\mathrm{rms}}\right)^{2} \delta\left(t-t^{\prime}\right),
\end{gathered}
$$

where no summation over double indices is assumed and the angle brackets mean here a temporal or ensemble average. For solving equation (C1) we use the Ansatz

$$
\overline{\boldsymbol{A}}(z, t)=\tilde{\boldsymbol{A}}(t) \exp (i k z)
$$

with an arbitrary, but fixed, wavenumber $k$ and employ a third-order Runge-Kutta time-stepping scheme. At each time step of length $\delta t$, the fluctuations of $\alpha_{i j}$ and $\eta_{i j}$ are taken as random numbers from a Gaussian distribution and scaled by $1 /(\delta t)^{1 / 2}$ so that equations (C3) and (C4) hold.

We recall that, if $\alpha_{i j}=\alpha \delta_{i j}$ with $\alpha=$ const $\ll S / k$, the critical value of the dynamo number $D_{\alpha S}$, as defined in equation (32) but with $\alpha^{\text {rms }}$ replaced by $\alpha$, is $D_{\alpha S}^{\text {crit }}=2$ (e.g., Brandenburg \& Subramanian 2005a). In the case of a pure incoherent alpha-shear dynamo, i.e., $D_{\eta S}=0$, it is found that $D_{\alpha S}^{\text {crit }} \approx 2.3$ (see the left panel of Fig. 13). On the other hand, for $D_{\alpha S}=0$ we have $D_{\eta S}^{\text {crit }} \approx 6.5$.

There are reversals on a typical timescale of about one diffusion time. However, this time can increase significantly if magnetic helicity conservation (appropriate for a closed domain) is taken into account (Field \& Blackman 2002; Blackman \& Brandenburg 2002; Subramanian 2002). This means that the $\alpha$ effect has to be amended by an additional term that results from the current helicity produced by the dynamo. We assume again $\alpha_{i j}=\alpha \delta_{i j}$ and a nonfluctuating $\eta_{i j}=\eta_{t} \delta_{i j}$, further

$$
\alpha(t)=\alpha_{K}(t)+\alpha_{M}(t)
$$

where $\alpha_{K}(t)$ is stochastic, just like $\alpha_{i j}(t)$ in equation (C3), and $\alpha_{M}(t)$ obeys the differential equation

$$
\frac{d \alpha_{M}}{d t}=-2 \eta_{t} k_{f}^{2}\left(\frac{\left|\tilde{\mathcal{E}}^{*} \cdot \tilde{\boldsymbol{B}}\right|}{B_{\mathrm{eq}}^{2}}+\frac{\alpha_{M}}{\mathrm{Re}_{M}}\right)
$$

with $\tilde{\boldsymbol{B}}$ and $\tilde{\mathcal{E}}$ defined analogously to $\tilde{\boldsymbol{A}}$ in equation (C5). The dynamo number $D_{\alpha S}$ is now defined with respect to $\alpha_{K}^{\text {rms }}$. Equation $(\mathrm{C} 7)$ is solved simultaneously with equation $(\mathrm{C} 1)$ using the aforementioned time-stepping scheme. As here a nonlinearity is introduced, the Ansatz from equation (C5) has now to be understood as a one-mode truncation. The model calculations show that the timescale for reversals increases proportional to $\mathrm{Re}_{M}^{1 / 2}$ (see the right panel of Fig. 13). 
Blackman, E. G., \& Brandenburg, A. 2002, ApJ, 579, 359

Brandenburg, A. 2001, ApJ, 550, 824

$$
\text { 2005a, ApJ, 625, } 539
$$$$
\text { 2005b, Astron. Nachr., 326, } 787
$$

Brandenburg, A., Jennings, R. L., Nordlund, Å., Rieutord, M., Stein, R. F., \& Tuominen, I. 1996, J. Fluid Mech., 306, 325

Brandenburg, A., Nordlund, Å., Stein, R. F., \& Torkelsson, U. 1995, ApJ, 446, 741

Brandenburg, A., Rädler, K.-H., \& Schrinner, M. 2008, A\&A, submitted (arXiv: 0801.1320)

Brandenburg, A., \& Subramanian, K. 2005a, Phys. Rep., 417, 1 2005b, A\&A, 439, 835

2005c, Astron. Nachr., 326, 400

Cattaneo, F. 1999, ApJ, 515, L39

Elperin, T., Kleeorin, N., \& Rogachevskii, I. 2003, Phys. Rev. E, 68, 016311

Fedotov, S., Bashkirtseva, I., \& Ryashko, L. 2006, Phys. Rev. E, 73, 066307

Field, G. B., \& Blackman, E. G. 2002, ApJ, 572, 685

Glatzmaier, G. A., \& Roberts, P. H. 1995, Nature, 377, 203

Hawley, J. F., Gammie, C. F., \& Balbus, S. A. 1995, ApJ, 440, 742

Hoyng, P. 1993, A\&A, 272, 321

Kraichnan, R. H. 1976, J. Fluid Mech., 75, 657

Krause, F., \& Rädler, K.-H. 1980, Mean-field Magnetohydrodynamics and Dynamo Theory (Oxford: Pergamon)

Meneguzzi, M., Frisch, U., \& Pouquet, A. 1981, Phys. Rev. Lett., 47, 1060

Meneguzzi, M., \& Pouquet, A. 1989, J. Fluid Mech., 205, 297

Moffatt, H. K. 1978, Magnetic Field Generation in Electrically Conducting Fluids (Cambridge: Cambridge Univ. Press), 175
REFERENCES

Nordlund, Å., Brandenburg, A., Jennings, R. L., Rieutord, M., Ruokolainen, J., Stein, R. F., \& Tuominen, I. 1992, ApJ, 392, 647

Piddington, J. H. 1981, ApJ, 247, 293

Proctor, M. R. E. 2007, MNRAS, 382, L39

Rädler, K.-H. 1969, Monats. Dt. Akad. Wiss., 11, 194 1980, Astron. Nachr., 301, 101

Rädler, K.-H., \& Stepanov, R. 2006, Phys. Rev. E, 73, 056311

Rogachevskii, I., \& Kleeorin, N. 2003, Phys. Rev. E, 68, 036301 . 2004, Phys. Rev. E, 70, 046310

Rüdiger, G., \& Kitchatinov, L. L. 2006, Astron. Nachr., 327, 298

Schrinner, M., Rädler, K.-H., Schmitt, D., Rheinhardt, M., \& Christensen, U. 2005, Astron. Nachr., 326, 245

2007, Geophys. Astrophys. Fluid Dyn., 101, 81

Silant'ev, N. A. 2000, A\&A, 364, 339

Sokolov, D. D. 1997, Astron. Rep., 41, 68

Subramanian, K. 2002, Bull. Astron. Soc. India, 30, 715

Sur, S., Brandenburg, A., \& Subramanian, K. 2008, MNRAS, in press (arXiv: 0711.3789)

Vainshtein, S. I., \& Cattaneo, F. 1992, ApJ, 393, 165

Vishniac, E. T., \& Brandenburg, A. 1997, ApJ, 475, 263

Vishniac, E. T., \& Cho, J. 2001, ApJ, 550, 752

Wisdom, J., \& Tremaine, S. 1988, AJ, 95, 925

Yousef, T. A., Heinemann, T., Schekochihin, A. A., Kleeorin, N., Rogachevskii, I., Iskakov, A. B., Cowley, S. C., \& McWilliams, J. C. 2007, Phys. Rev. Lett., submitted (arXiv: 0710.3359) 